\title{
The hybrid disks: a search and study to better understand evolution of disks ${ }^{\star}$
}

\author{
J. Péricaud ${ }^{1,2}$, E. Di Folco ${ }^{1,2}$, A. Dutrey ${ }^{1,2}$, S. Guilloteau ${ }^{1,2}$, and V. Piétu ${ }^{3}$ \\ 1 Univ. Bordeaux, LAB, UMR 5804, 33615 Pessac, France \\ e-mail: jpericaud@outlook.fr \\ 2 CNRS, LAB, UMR 5804, 33615 Pessac, France \\ 3 IRAM, 300 rue de la piscine, 38406 Saint-Martin-d'Hères, France
}

Received 22 July 2016 / Accepted 1 December 2016

\begin{abstract}
Context. The increased sensitivity of millimeter-wave facilities now makes possible the detection of low amounts of gas in debris disks. Some of the gas-rich debris disks harbor peculiar properties, with possible pristine gas and secondary generated dust. The origin of the gas in these hybrid disks is strongly debated and the current sample is too sparse to understand this phenomenon.

Aims. More detections are necessary to increase the statistics on this population. Lying at the final stages of evolution of protoplanetary disks and at the beginning of the debris disk phase, these objects could provide new insight into the processes involved in the making of planetary systems.

Methods. We carried out a deep survey of the CO $J=2 \rightarrow 1$ and CO $J=3 \rightarrow 2$ lines with the APEX and IRAM radiotelescopes in young debris disks selected according to hybrid disk properties. The survey is complemented with a bibliographic study of the ratio between the emission of the gas and the continuum $\left(S_{\mathrm{CO}} / F_{\text {cont }}\right)$ in CTTS, Herbig Ae, WTTS, hybrid, and debris disks.

Results. Our sub-mm survey comprises 25 stars, including 17 new targets, and we increase the sensitivity limit by a factor 2 on eight sources compared to similar published studies. We report a $4 \sigma$ tentative detection of a double-peaked CO $J=2 \rightarrow 1$ line around HD 23642; an eclipsing binary located in the Pleiades. We also reveal a correlation between the emission of the CO gas and the dust continuum from CTTS, Herbig Ae and few debris disks. The observed trend of the gas to dust flux ratio suggests a concurrent dissipation of the dust and gas components. Hybrid disks systematically lie above this trend, suggesting that these systems may witness a transient phase, when the dust has evolved more rapidly than the gas, with a flux ratio $S_{\mathrm{Co}} / F_{\text {cont }}$ enhanced by a factor of between 10 and 100 compared to standard (proto-)planetary disks.
\end{abstract}

Key words. circumstellar matter - protoplanetary disks - radio lines: stars

\section{Introduction}

The scenarios of planet formation in disks are constrained by the observed lifetime of circumstellar material around pre-main sequence stars. This is particularly true for the late accretion phases of giant gaseous planets.

In gas-rich disks, significant progress has been made in the past decade to find direct evidence for disk evolution. Inner holes have been found in an increasing number of proto-planetary Class II disks (e.g. LkCa15, Piétu et al. 2006), with a frequency as high as $20 \%$ (e.g. Owen \& Clarke 2012). In the cavity, the evolution of the gaseous component, and how it can affect the dynamics of the small grains during the last stages of (giant) planet formation is not yet known. Near-IR images of these transitional disks revealed for instance smaller inner holes than those seen at millimeter wavelengths (Garufi et al. 2013), a possible consequence of the spatial distribution of the residual, primordial gas.

On the other hand, significant IR excess has been associated with many main sequence stars (e.g. Eiroa et al. 2013), without any significant reservoir of gas except for a few younger ( $<100$ Myr) stars (Dent et al. 2013; Howard et al. 2013). In these systems, the dust lifetime is short, and therefore the observed

\footnotetext{
* Reduced data used in the paper (FITS) are only available at the CDS via anonymous ftp to cdsarc.u-strasbg. fr (130.79.128.5) or via http://cdsarc.u-strasbg.fr/viz-bin/qcat?J/A+A/600/A62
}

dust must be constantly replenished from collisions between exo-asteroids and/or exo-comets. These so-called debris disks represent extra-solar analogs to our Kuiper Belt. The young $\beta$ Pictoris system is an impressive example of this sub-category. It has an optically thin dust disk, and ALMA images of the cold $\mathrm{CO}$ emission reveal asymmetries that are attributed to the destruction of cometary-like bodies, possibly trapped by an unseen planet (Dent et al. 2014). The gas in this system is therefore not primordial.

Among young MS stars surrounded by debris disks, a handful of remarkable objects exhibit a substantial amount of primordial gas, making them hybrid disks between proto-planetary and debris disks: 49 Ceti (40 Myr, Zuckerman et al. 1995; Moór et al. 2011), HD 21997 (30 Myr, Kóspál et al. 2013), HD 141569 (5 Myr, Dent et al. 2005), and HD 131835 (16 Myr, Moór et al. 2015). This raises questions over the specific physical conditions that allow $\mathrm{CO}$ gas disks to survive in stars as old as $30 \mathrm{Myr}$ since most disks dissipate in $\sim 5-10 \mathrm{Myr}$ only. The dominant physical mechanisms at play that dissipate such old gas disks are photoevaporation or runaway gas accretion by unseen giant planets, which may then present different characteristics since they would accrete on a longer timescale. The paucity of such hybrid systems may be explained by the very fast dissipation of the primordial reservoir of gas, as soon as this process has started. They may represent the missing link 
Table 1. Observational parameters for our sample of debris disks.

\begin{tabular}{|c|c|c|c|c|c|c|}
\hline Name & $\begin{array}{l}\text { LSR velocity } \\
\left(\mathrm{km} \mathrm{s}^{-1}\right)\end{array}$ & $L_{\mathrm{IR}} / L_{\star}$ & Ref. & $\begin{array}{c}3 \sigma_{\text {int }} \\
\left(\mathrm{Jy} \mathrm{km} \mathrm{s}^{-1}\right)\end{array}$ & 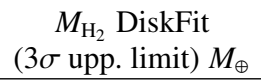 & $\begin{array}{c}M_{\mathrm{H}_{2}} \text { from } S_{\mathrm{CO}} \\
(3 \sigma \text { upp. limit }) M_{\oplus}\end{array}$ \\
\hline \multicolumn{7}{|c|}{ IRAM-30 $\mathrm{m}-\mathrm{CO} J=2 \rightarrow 1$} \\
\hline HD 2772 & -5.4 & $8.2 \times 10^{-6}$ & 1 & $<0.569$ & $<0.110$ & $<0.454$ \\
\hline HD 14055 & 7.5 & $6.7 \times 10^{-5}$ & 1 & $<0.387$ & $<0.008$ & $<0.027$ \\
\hline$H D 15115$ & -0.2 & $4.8 \times 10^{-4}$ & 2 & $<1.89$ & $<0.063$ & $<0.229$ \\
\hline HD 21620 & -22.8 & $2.51 \times 10^{-5}$ & 3 & $<0.622$ & $<0.383$ & $<0.819$ \\
\hline HD 23642 & -4.0 & $2.2 \times 10^{-5}$ & 1 & $0.71 \pm 0.18$ & $0.18 \pm 0.04$ & $0.57 \pm 0.14$ \\
\hline HD 31295 & -3.5 & $4.7 \times 10^{-5}$ & 1 & $<0.768$ & $<0.016$ & $<0.058$ \\
\hline HD 42111 & 8.4 & $1.00 \times 10^{-5}$ & 3 & $<0.902$ & $<1.02$ & $<2.16$ \\
\hline HD 159082 & 7.1 & $5.7 \times 10^{-5}$ & 1 & $<0.497$ & $<0.132$ & $<0.537$ \\
\hline HD 218396 & -12.6 & $2.29 \times 10^{-4}$ & 4 & $<0.349$ & $<0.005$ & $<0.032$ \\
\hline \multicolumn{7}{|c|}{$\mathrm{APEX}-\mathrm{CO} J=3 \rightarrow 2$} \\
\hline HD 225200 & 13.4 & $7.4 \times 10^{-5}$ & 1 & $<3.15$ & $<0.260$ & $<0.470$ \\
\hline HD 17848 & 16.7 & $4.8 \times 10^{-5}$ & 1 & $<10.5$ & $<0.146$ & $<0.242$ \\
\hline HD 24966 & 11.7 & $3.1 \times 10^{-4}$ & 1 & $<2.85$ & $<0.166$ & $<0.278$ \\
\hline HD 30422 & -4.8 & $3.7 \times 10^{-5}$ & 1 & $<2.22$ & $<0.036$ & $<0.063$ \\
\hline HD 35850 & 1.4 & $2.3 \times 10^{-5}$ & 1 & $<2.26$ & $<0.007$ & $<0.015$ \\
\hline HD 38206 & 5.8 & $1.4 \times 10^{-4}$ & 1 & $<2.78$ & $<0.062$ & $<0.119$ \\
\hline HD 54341 & $20.0^{*}$ & $1.9 \times 10^{-4}$ & 1 & $<2.49$ & $<0.106$ & $<0.232$ \\
\hline HD 71043 & 5.9 & $6.5 \times 10^{-5}$ & 1 & $<2.74$ & $<0.073$ & $<0.131$ \\
\hline HD 71155 & -4.7 & $4.2 \times 10^{-5}$ & 1 & $<2.66$ & $<0.017$ & $<0.035$ \\
\hline HD 78072 & 2.5 & $2.5 \times 10^{-4}$ & 1 & $<2.11$ & $<0.071$ & $<0.133$ \\
\hline$H D 136246$ & 4.2 & $4.9 \times 10^{-5}$ & 1 & $<2.47$ & $<0.281$ & $<0.459$ \\
\hline HD 164249 & 2.5 & $7.2 \times 10^{-4}$ & 1 & $<2.38$ & $<0.020$ & $<0.049$ \\
\hline HD 166191 & 3.8 & $1.0 \times 10^{-1}$ & 5 & $<2.77$ & $<0.187$ & $<0.351$ \\
\hline HD 181296 & 14.3 & $2.4 \times 10^{-4}$ & 1 & $<2.19$ & $<0.014$ & $<0.046$ \\
\hline HD 182681 & 10.5 & $1.95 \times 10^{-4}$ & 4 & $<2.91$ & $<0.083$ & $<0.124$ \\
\hline HD 220825 & -1.5 & $2.9 \times 10^{-5}$ & 1 & $<2.29$ & $<0.011$ & $<0.046$ \\
\hline
\end{tabular}

Notes. ${ }^{(*)}$ The radial velocity of HD 54341 was kindly provided by F. Kiefer, see Kiefer (in prep.). Stars in italics are systems with earlier detection limits in one $\mathrm{CO}$ transition, see Sect. 2.1. The last three colums show the $3 \sigma$ upper limits derived from our study: on the integrated intensity of $\mathrm{CO}$, the $\mathrm{H}_{2}$ mass derived from DiskFit model and the $\mathrm{H}_{2}$ mass directly derived from the integrated flux. The integrated flux and the $\mathrm{H}_{2}$ masses for the tentative detection around HD 23642 are also displayed.

References. For $L_{\mathrm{IR}} / L_{\star}$ : 1 - Chen et al. (2014); 2 - Moór et al. (2011); 3 - Roberge \& Weinberger (2008); 4 - Rhee et al. (2007); 5 Schneider et al. (2013).

between proto-planetary and planetary systems, as suggested by the recent analysis of the ALMA observations of HD 21997 by Kóspál et al. (2013), and should therefore be considered as transient systems with ongoing dissipation of their primordial gas component and last stages of (giant) planet formation. However, in some cases at least, part of the observed CO gas may be of second generation as suggested for 49 Ceti (Roberge et al. 2013). So far, all these disks have been found around Herbig Ae stars and there are no equivalent hybrid disks observed around old T Tauri stars (Lieman-Sifry et al. 2016).

Millimeter surveys of gas in debris disks yielded low detection rates, confirming the paucity of gas-rich disks. Dent et al. (2005) reported the first detection of CO $J=3 \rightarrow 2$ in 49 Ceti from JCMT observations out of a sample of $59 \mathrm{HAeBe}$ and Vega-like stars. Pascucci et al. (2006) focused on 15 (5-100 Myr old) stars from the FEPS sample, but their SMT search for CO $J=2 \rightarrow 1$ and $J=3 \rightarrow 2$ lines yielded only non detections and upper-limits on the gas mass. Kastner et al. (2010) also failed to detect molecular gas emission with IRAM-30 m antenna in a sample of 10 Vega-like stars. Only recently, Moór et al. (2011) discovered a significant CO $J=3 \rightarrow 2$ and CO $J=2 \rightarrow 1$ emission from the 30 Myr old star HD 21997 using the APEX/SHFI receiver. The most sensitive survey, however, only reached upper limits on the $\mathrm{CO}$ gas mass of the order of $M_{\mathrm{CO}} \lesssim 0.5-1 \times 10^{-3} M_{\oplus}$.
We report here a more sensitive survey of 25 debris disks in CO $J=2 \rightarrow 1$ or $J=3 \rightarrow 2$ using APEX and the IRAM-30 m radiotelescope. With these observations, we reach a sensitivity limit similar to that of the recent work of Moór et al. (2015). We have also complemented these new observations with a compilation of more recent observations of dust and gas in young disks. We report the results in this paper. Section 2 outlines the sample of sources and the observations. The description of the analysis by correlation is given in Sect. 3 while Sect. 4 is an open discussion of these results.

\section{Samples and data reduction}

\subsection{APEX and IRAM observations}

We performed a ${ }^{12} \mathrm{CO} J=3 \rightarrow 2$ and ${ }^{12} \mathrm{CO} J=2 \rightarrow 1$ survey of the sample presented in Table 1 . We selected 25 systems whose properties are similar to the recently identified gasrich debris disks, thereby focusing on A-type stars younger than $100 \mathrm{Myr}$ and with a large dust radius $R_{\text {dust }}>30$ au. The sources were collected from various IRAS, Spitzer, WISE, and Herschel debris disks studies (Rhee et al. 2007; Wyatt et al. 2007; Zuckerman \& Song 2004; Patel et al. 2014; Morales et al. 2009; Chen et al. 2006; Riviere-Marichalar et al. 2014; Kennedy et al. 2014). Among the 25 selected targets, 17 are new sources for 


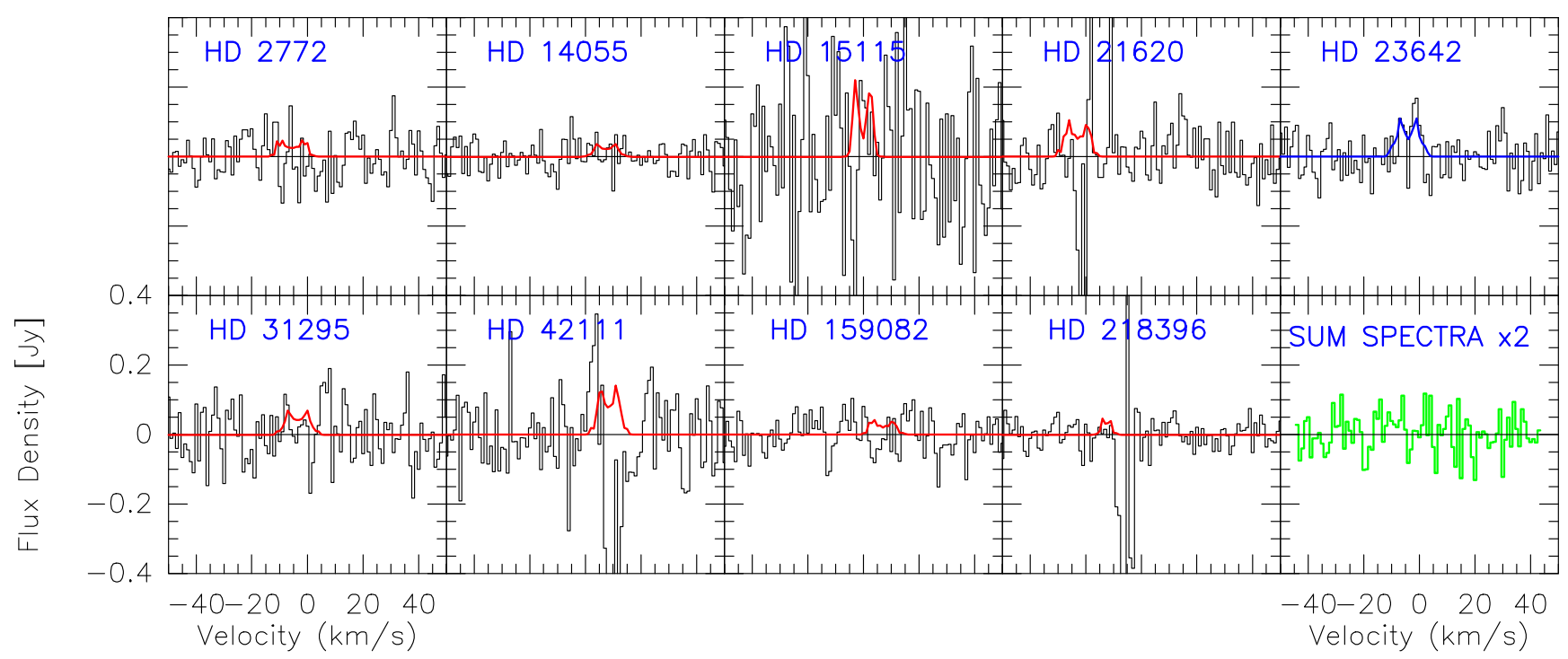

Fig. 1. CO $J=2 \rightarrow 1$ spectra obtained with the IRAM-30 $\mathrm{m}$ telescope. Fluxes are in Jy with respect to local standard of rest (LSR) velocity. The last box shows the cumulated spectrum made with spectra shifted in velocity to set the systemic velocity to zero (note that the flux scale is different from the individual spectra). The sources with a high rms or showing strong contamination (i.e., HD 15115, HD 21620, HD 42111 and HD 218396) are not used to produce the cumulated spectrum. The $3 \sigma$ upper limit model for each spectrum is over-plotted in red (see sect. 3.3) except for the HD 23642 spectrum, where the best-fit model for the tentative detection is plotted in blue.

the search of $\mathrm{CO}$ and 8 sources had been observed in only one of these two CO transitions. We also included 2 targets with atomic gas sprectroscopic features interpreted as the signature of falling-evaporating bodies (FEB; HD 21620 and HD 42111, Welsh \& Montgomery 2013).

For northern targets, we used the IRAM-30 m telescope (project 172-14), attempting to detect the CO $J=2 \rightarrow 1$ line with the EMIR receiver connected to the FTS200 backend providing $200 \mathrm{kHz}$ spectral resolution. In the southern hemisphere, we used the APEX telescope at $345 \mathrm{GHz}$ to search for the $\mathrm{CO} J=3 \rightarrow 2$ transition with the SHeFI receiver (projects 094.C-0161 and 095.C-0742).

The data reduction was done using the GILDAS/CLASS package. Spectra have been resampled to a $\delta v=1 \mathrm{~km} \mathrm{~s}^{-1}$ channel width. The baseline subtraction and rms calculation were performed on 800 channels, avoiding the contamination lines when present. The conversion from the $T_{\mathrm{A}}^{*}$ scale onto flux density (Jy) was done with the following factors: $41 \mathrm{Jy} / \mathrm{K}$ for APEX ${ }^{1}$ at $345 \mathrm{GHz}$ and $7.8 \mathrm{Jy} / \mathrm{K}$ for the IRAM-30 $\mathrm{m}^{2}$ at $230 \mathrm{GHz}$. The final spectra are plotted in Figs. 1 and 2, and the upper limits on $\mathrm{CO}$ emission are displayed as a function of the infrared excess of disks in Fig. 3. The median distance of the observed stars is $69 \mathrm{pc}$, with a standard deviation of $46 \mathrm{pc}$. Since the flux values depend on the distance of the stars, they are not directly representative of the gas mass reservoir. We therefore present the fluxes scaled at $100 \mathrm{pc}$ for all 25 stars in our sample and other targets from the literature in Fig. 4.

\subsection{Compilation of existing $\mathrm{CO}$ and $\mathrm{mm}$ continuum data}

We have compiled measurements of the $\mathrm{CO} J=2 \rightarrow 1$ and $\mathrm{CO} J=3 \rightarrow 2$ emission from the literature, as well as the continuum emission at the corresponding wavelength for a wide variety of disks. We consider five categories: the classical T Tauri stars

\footnotetext{
1 http://www.apex-telescope.org/telescope/efficiency/

2 http://www.iram.es/IRAMES/mainWiki/

Iram30mEfficiencies/
}

(CTTS), the Herbig AeBe, the weak-line T Tauri stars (WTTS), the hybrid disks, and finally the debris disks. We do not include class I objects in the sample to avoid confusion with remnant envelopes and nearby molecular clouds. The measurements are compiled in Table D.1. We have retrieved the integrated emission $\left(\mathrm{Jy} \mathrm{km} \mathrm{s}^{-1}\right)$ for the $\mathrm{CO}$ lines, and the upper limits are always given at the $3 \sigma$ level.

We have also included binary systems. As most of them are either spectroscopic or wide binaries, the dynamical influence on the disk should be negligible. We selected binary sources from the ALMA survey of Akeson \& Jensen (2014). The authors focussed on the analysis of the continuum emission, and we have retrieved the $\mathrm{CO} J=3 \rightarrow 2$ emission of 15 sources from the archive (project 2011.0.00150.S). We included binaries with dynamical truncation, such as GG Tau, since no systematic effect on the ratio $S_{\mathrm{CO}} / F_{\text {cont }}$ is seen. We have also used the data from the ALMA project 2013.1.00163.S (PI: M. Simon), which aims at determining the stellar mass of low-mass T Tauri stars by measuring the Keplerian rotation of the gas. This project adds eight sources to our compilation. In addition, for HD 21997, we have determined the upper limit on the dust emission at $1.3 \mathrm{~mm}$ from the archival data (project 2011.0.00780.S).

Most of these sources are in young stellar associations. The most represented complex is the Taurus-Auriga region, with approximatively half of the CTTS and Herbig AeBe, and one third of WTTS. Other stars are either isolated or belong to different clouds such as Ophiucus, upper Scorpius, TW Hya, Orion, Chameleon.

The measurements at $1.3 \mathrm{~mm}$ or $0.87 \mathrm{~mm}$ are not available for every source. However, we find a clear correlation between the observed CO $J=2 \rightarrow 1 / \mathrm{CO} J=3 \rightarrow 2$ fluxes (scaled at $100 \mathrm{pc}$ ). We have thus tried to complement the missing measurements by interpolating the flux of the missing transition on this correlation line (the procedure is described in Appendix B). This assumes that the disks have physical conditions similar to those used to derive the correlation. The corresponding fluxes are represented by smaller symbols in Figs. 7a and b. 


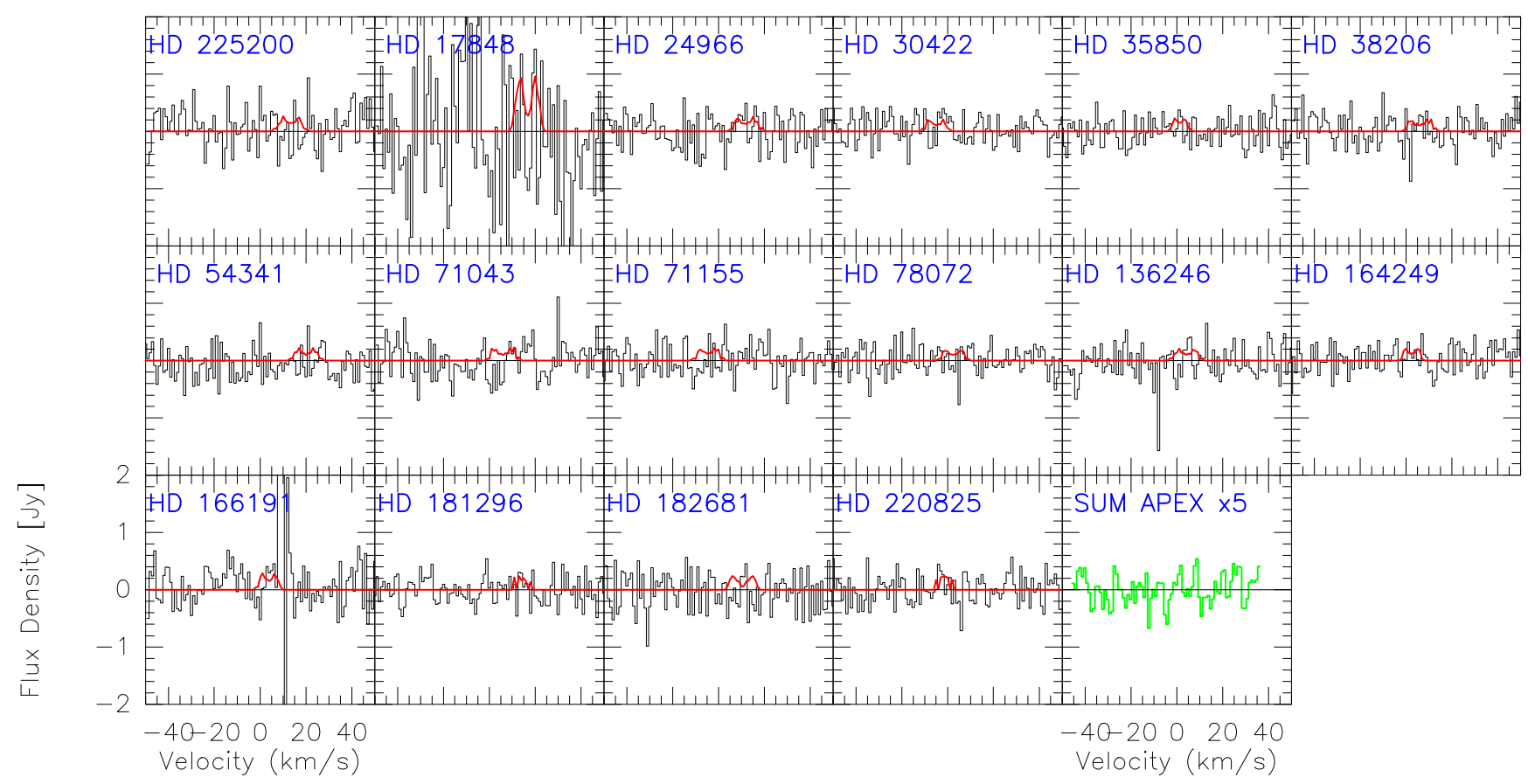

Fig. 2. CO $J=3 \rightarrow 2$ spectra obtained with the APEX telescope. Fluxes are in Jy with respect to LSR velocity. The last box shows the cumulated spectrum made with spectra shifted in velocity to set the systemic velocity to zero (multiplied by factor five). The sources with a high rms or showing strong contamination (i.e., HD 17848 and HD 166191) are not used to produce the cumulated spectrum. The $3 \sigma$ upper limit model for each spectrum is over-plotted in red (see sect. 3.3).

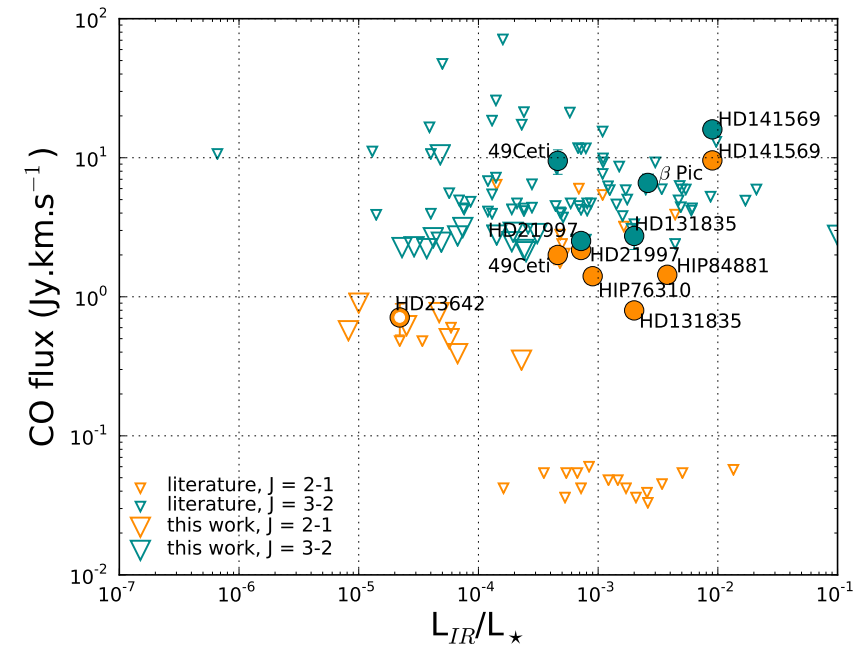

Fig. 3. CO line flux as a function of infrared excess. Circles indicate $\mathrm{CO}$ detection, and triangles indicate upper limits. Large symbols are from our survey, and small symbols for literature data (Dent et al. 2005; Kastner et al. 2010; Moór et al. 2011, 2015; Hales et al. 2014; Lieman-Sifry et al. 2016). Orange symbols stand for the CO $J=2 \rightarrow 1$ measurements and green symbols for $\mathrm{CO} J=3 \rightarrow 2$ measurements. The tentative detection around HD 23642 is plotted with an empty circle.

\section{Results and analysis}

\subsection{Outcome of the APEX and IRAM survey}

Our IRAM and APEX surveys were designed to provide a homogeneous sensitivity for the whole sample, and to reach a highenough sensitivity on the integrated intensity to detect dim systems such as 49 Ceti at a $>5 \sigma$ level. This corresponds, for a channel width of $1 \mathrm{~km} \mathrm{~s}^{-1}$, to a $6 \mathrm{mK} \mathrm{rms} \mathrm{value} \mathrm{for} T_{\mathrm{A}}^{*}$ with

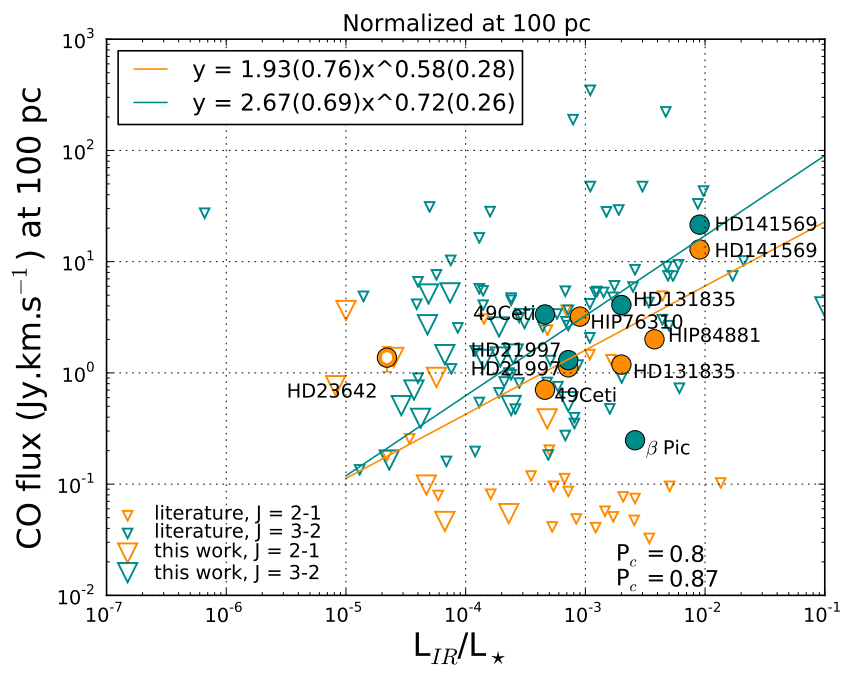

Fig. 4. Integrated flux of CO normalized at $100 \mathrm{pc}$ compared to the infrared fractional luminosity of the debris disks. The same disks and symbol/color code are used as in Fig. 3.

APEX and $6.5 \mathrm{mK}$ with the IRAM-30 m telescope. Most targets present no $\mathrm{CO}$ line detection, and we report the $3 \sigma_{\text {int }}$ upper limits on the integrated intensity, with the standard formula $\sigma_{\text {int }}=\sigma \cdot \sqrt{\Delta v \delta v}, \sigma$ being the intensity rms in a single channel. Although typical line widths of approximately $5 \mathrm{~km} \mathrm{~s}^{-1}$ are observed around A-stars with detected CO lines, we assume here $\delta v=10 \mathrm{~km} \mathrm{~s}^{-1}$ for the sake of comparison with previous studies. Contamination from the cloud environment is seen for five sources: HD 218396, HD 42111, HD 21620, HD 166191, and HD 136246. For each CO transition, we also stacked the spectra of all observed targets, after correcting for the known system velocities, in order to improve the global signal-to-noise ratio, but 


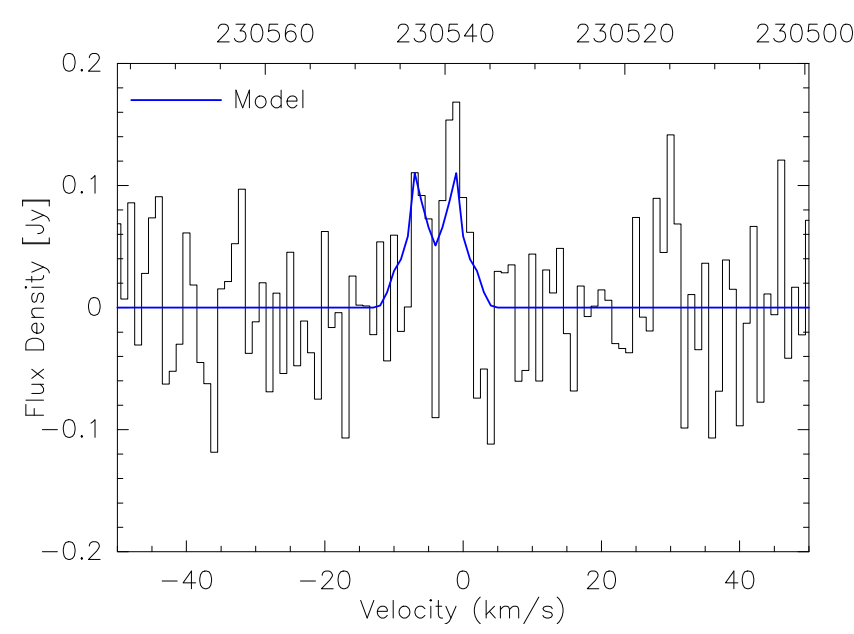

Fig. 5. CO $J=2 \rightarrow 1$ spectrum of HD 23642 obtained with the IRAM$30 \mathrm{~m}$ telescope. Flux is in Jy with respect to LSR velocity. A line is detected at the $4 \sigma$ level, at the star velocity $\left(-3.6 \mathrm{~km} \mathrm{~s}^{-1}\right)$. The model of the line is displayed in blue.

this did not yield any tentative detection. The stacked spectra are displayed in green in the last insets of Figs. 1 and 2. In Table 1, we present the flux density upper limits for seven new targets in the $\mathrm{CO} J=2 \rightarrow 1$ transition, and two stars that had already been targeted in the CO $J=3 \rightarrow 2$ transition (namely, HD 15115 and HD 218396). The median value is $0.8 \mathrm{Jy} \mathrm{km} \mathrm{s}^{-1}\left(3 \sigma_{\text {int }}\right)$. With APEX, we report upper limits for ten new targets and improve the detection limits by a factor two at least for the other disks (median value $3 \sigma_{\text {int }}=2.6 \mathrm{Jy} \mathrm{km} \mathrm{s}^{-1}$ ), compared to earlier studies Kastner et al. (2010), Moór et al. (2011), Hales et al. (2014), except for HD 17848 where Moór et al. (2015) obtained more sensitive data.

\subsection{A possible new hybrid disk candidate in the Pleiades}

We report a tentative detection of $\mathrm{CO} J=2 \rightarrow 1$ for the doublelined eclipsing binary HD $23642(\mathrm{~A} 0 \mathrm{Vp}(\mathrm{Si})+\mathrm{Am}, P=2.46 \mathrm{~d}$, Abt \& Levato 1978; Torres 2003; Munari et al. 2004). The system is classified as a debris disk with a weak infrared excess detected by Spitzer ( $\mathrm{Su}$ et al. 2006; Morales et al. 2009; Chen et al. 2014). The peak emission is found around $24 \mu \mathrm{m}$, although there is only an upper limit on the flux at $70 \mu \mathrm{m}$. Here we adopt the fractional excess luminosity reported by Morales et al. (2009), $L_{\mathrm{IR}} / L_{\star}=1.7 \times 10^{-5}$, which relies on the shape of the IRS spectrum in the $20-33 \mu \mathrm{m}$ range. The binary is composed of two A-type stars, located in the Pleiades group. Its distance has been revised to $138 \pm 1.5 \mathrm{pc}$ by Groenewegen et al. (2007), in agreement with the recent works on the distance of the Pleiades.

The spectrum (see Fig. 5) presents a double-peaked line, with an intensity integrated over the channels -8 to $+2 \mathrm{~km} \mathrm{~s}^{-1}$ estimated to $710 \pm 180 \mathrm{mJy} \mathrm{km} \mathrm{s}^{-1}$. The line center is at $\sim-4 \mathrm{~km} \mathrm{~s}^{-1}$, consistent with the binary radial velocity of $V_{\mathrm{LSR}}=-3.6 \pm$ $0.1 \mathrm{~km} \mathrm{~s}^{-1}$ determined by Groenewegen et al. (2007). This signal is unlikely to be due to contamination by a molecular cloud emission: although $\mathrm{CO}$ emission is present towards the Pleiades region, it appears at LSR velocities of $\sim 7$ and $10 \mathrm{~km} \mathrm{~s}^{-1}$ (Breger 1987; White 2003).

If we interpret this signal as emission from a Keplerian disk, given the total stellar mass derived from spectroscopic data $\left(2.23 M_{\odot}\right.$ and $1.57 M_{\odot}$ ), and the inclination set to $78^{\circ}$ (e.g., Groenewegen et al. 2007), the separation of the two peaks in the

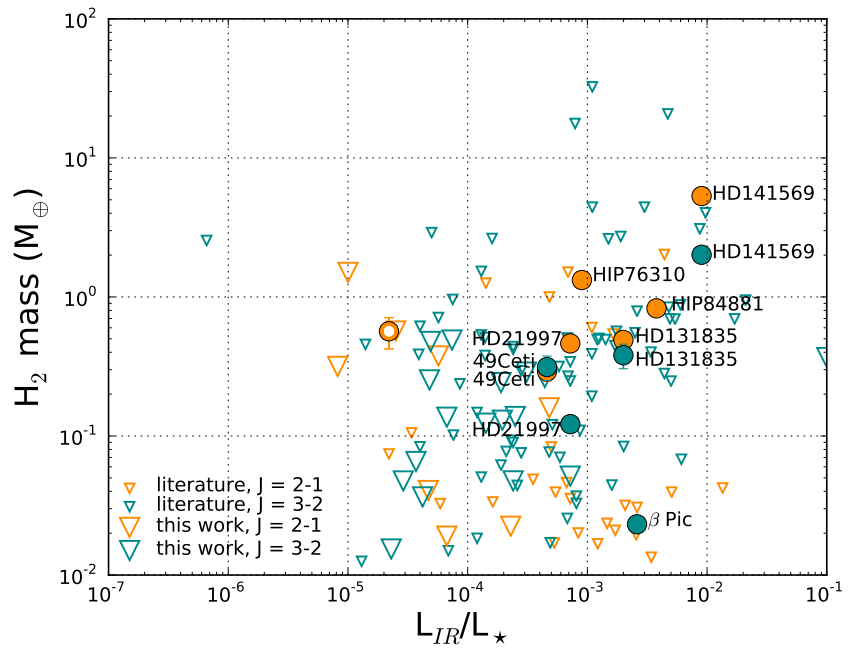

Fig. 6. Mass of $\mathrm{H}_{2}$ (from Scoville et al. 1986) compared to the infrared fractional luminosity of the debris disks. The same disks and symbol/color code are used as in Fig. 3.

CO line suggests a disk outer radius much larger than $200 \mathrm{au}$. Using the DiskFit tool (see Sect. 3.3), the best-fit model yields $R_{\text {out }}=450 \pm 210 \mathrm{au}$, and a CO gas column density at $100 \mathrm{au}$ of $\Sigma_{0}=6.0 \times 10^{14} \pm 1.3 \times 10^{14} \mathrm{~cm}^{-2}(4.6 \sigma$ detection $)$. The best fit model is overplotted in Fig. 5.

The derived mass of gas in this system would be similar to that of the 20-40 Myr old systems HD 21997 and 49 Ceti, for which the gas origin is debated. Given the estimated age of the Pleiades cluster ( 125-130 Myr, Stauffer et al. 1998; Castellani et al. 2002), the presence of such a large amount of remnant gas is puzzling and makes it the oldest hybrid disk candidate. Further observations are necessary to definitely confirm our finding, however the double-peaked line shape centered at the expected stellar velocity suggests a robust detection.

\subsection{CO mass upper limits}

The upper limits on $\mathrm{CO}$ integrated emission can be translated into limits on the remnant mass of $\mathrm{H}_{2}$ gas. We derive the gas mass using two methods. The first is based on the rms obtained from the data. The second properly takes into account the velocity gradient resulting from Keplerian shear by using a disk model. Conversion to $\mathrm{H}_{2}$ content is done using the standard value of $10^{-4}$ for the $\mathrm{CO}$ to $\mathrm{H}_{2}$ abundance ratio.

Gas mass inferred from the rms of the flux density: here we follow the approach used by many authors and derive the CO content assuming $T_{\mathrm{ex}}=40 \mathrm{~K}$ for the excitation temperature and $\tau=1$ for the opacity (see Appendix A.1). These values allow a direct comparison with the literature (although a temperature $T_{\mathrm{ex}}=20 \mathrm{~K}$ is sometimes assumed). Results are shown in Table 1. Some sources have been observed in CO $J=2 \rightarrow 1$ and $3 \rightarrow 2$. The flux ratio and $\mathrm{H}_{2}$ masses derived from the two transitions are shown in Table B.1. The discrepancy in derived masses illustrates the limits of our assumptions. We note also that Kóspál et al. (2013) have derived excitation temperatures lower than $9 \mathrm{~K}$ for the ${ }^{12} \mathrm{CO}$ in the HD 21997 disk.

Figure 6 displays the total gas mass $\left(\mathrm{H}_{2}\right)$ consistently derived using this method as a function of the dust fractional luminosity, for sources from past surveys and from this work (larger symbols). The median value for the limits on the gas mass $\left(M_{\mathrm{H}_{2}}\right)$ in 
our sample is $0.1 M_{\oplus}(3 \sigma)$. The tightest constraint is obtained for HD 14055 in CO $J=2 \rightarrow 1$ and HD 35850 in CO $J=3 \rightarrow 2$ transition with a limit as low as $\sim 0.03 M_{\oplus}$, that is, $2 M_{\text {Moon }}$ of $\mathrm{H}_{2}$, a value equivalent to (even slightly less than) the amount of gas recently detected with ALMA in the young $\beta$ Pic system by Dent et al. (2014).

Gas mass inferred from a Keplerian disk model: the gas mass can also be estimated by constraining the surface density in a disk modeling approach, as done in Dutrey et al. (2011) and Chapillon et al. (2012). For this purpose, we used the DiskFit code (Piétu et al. 2007), dedicated to the simulation of Keplerian disks. We describe the radial physical properties of disks as power-laws, in a similar analysis to resolved disks. The disks are assumed to be in Keplerian rotation around their central stars, the mass of stars being estimated from their stellar types ${ }^{3}$. We assume for the disk model an outer radius $R_{\text {out }}=200$ au, a typical value for the resolved hybrid disks, and an inner radius $R_{\text {in }}=$ $5 \mathrm{au}$. For six disks, the inclination is known from resolved scattered light observations: $80^{\circ}$ for HD 182681, $73^{\circ}$ for HD 17848 (Moór et al. 2015), 56.7 ${ }^{\circ}$ for HD 71155 (Booth et al. 2013), $86^{\circ}$ for HD 15115 (Mazoyer et al. 2014), 26 ${ }^{\circ}$ for HD 218396 (Matthews et al. 2014). For HD 181296, we have set the inclination to the $20^{\circ}$ upper limit determined by Smith et al. (2009). The most probable value of $60^{\circ}$ is assumed for the other disks. The temperature law is set to $30 \mathrm{~K}$ at 100 au with the exponent of the radial variation $q=0.4$. With an assumed exponent of $p=1.5$ for the surface density (see Piétu et al. 2007, for case studies), the surface density at 100 au $\Sigma_{0}$ is the only free parameter.

The model spectra corresponding to the $3 \sigma$ upper limits on $\Sigma_{0}$ are superimposed in red to the spectra in Figs. 1 and 2. In addition, the best fit model for the tentative detection in HD 23643 is displayed in blue. The corresponding $3 \sigma$ upper limits on the total disk masses are reported in Table 1 (see Appendix A.2 for details). The derived gas masses are a factor of two or three lower than from the simple uniform $T_{\text {ex }}$ approach (see Table 1), as a result of different assumptions. As it accounts for density and temperature gradients, the method using DiskFit should be considered as more reliable.

\subsection{Detection statistics}

Our survey adds 17 new systems to the sample of young debris disks which have been searched for molecular gas. Taking into account these new constraints in addition to the surveys of Kastner et al. (2010), Moór et al. (2011), Hales et al. (2014), the recent survey of Moór et al. (2015) with the discovery of HD 131835, and the ALMA detections of gas around HIP 84881 and HIP 76310 in the survey of Lieman-Sifry et al. (2016), we can derive an approximate estimate of the occurrence of $\mathrm{CO}$ gas in young debris disks: $7.1 \pm 2.6 \%$ ( $1 \sigma$ uncertainty, 7 detections among 98 observed systems). This value can be considered as a lower limit since only less than half of the targets have been observed with enough sensitivity to detect a CO luminosity as large as that of HD 21997/49 Ceti (see Fig. 4). The occurrence of a CO gas disk as bright as HD 21997/49 Ceti (after scaling at $100 \mathrm{pc})$ is $12.2 \pm 4.7 \%(1 \sigma)(6 / 49$ detections $)$.

This value suggests that such systems are very rare, likely because they represent a very short transient phase in the evolution

\footnotetext{
3 http://www.uni.edu/morgans/astro/course/Notes/ section2/spectralmasses.html
}

of circumstellar disks. It should also be recognised that this detection frequency does not rely on a homogeneous sample: the observed targets are located in different regions of the sky, may span a broad range of ages, and the various surveys have reached different sensitivity limits. The occurrence of hybrid disks (with secondary dust and possible primordial CO gas) is even smaller, since the origin of the detected CO gas is still debated for most gas-rich debris disks (HD 141569, HD 21997, 49 Ceti and HD 131835). Several authors favor a secondary origin (Moór et al. 2011; Zuckerman \& Song 2012) for some of these systems, as is the case for the famous $\beta$ Pictoris disk, where the $\mathrm{CO}$ gas detected by ALMA may have been produced by a massive collision of planetesimals (Dent et al. 2014). Besides $\beta$ Pic, the detected systems with the weaker $\mathrm{CO}$ emission (or gas mass) are HD 21997 and 49 Ceti (see Figs. 4 and 6). These two are therefore the most probable candidates for a secondary origin of the gas, although the gas mass remains one order of magnitude larger than in $\beta$ Pic.

Among the stars in our study, we note that 17 disks (45 when including the surveys from the literature), that is, $68 \%$ of our sample (resp. 48\%), are constrained to CO gas masses smaller than that of 49 Ceti, and one of them, HD 35850, shows even less CO gas than $\beta$ Pictoris.

\subsection{CO to dust flux ratio correlations}

To better understand the peculiarity of hybrid disks, we compare their properties with those of other (proto-planetary) disks. For this purpose, we have chosen to study the ratio of the emission of $\mathrm{CO}$ over the emission of the dust, $\frac{S_{\mathrm{CO}}}{F_{\mathrm{cont}}}$. In the literature, we find many studies considering the ratio of masses, but the mass is always a calculated value, depending on models and on assumptions about excitation conditions and dust-to-mass ratio, etc. On the contrary, the $\frac{S_{\mathrm{CO}}}{F_{\text {cont }}}$, which is an equivalent width, is a distanceindependent product of observations that is not affected by hidden assumptions or biased by interpretation models. It may be impacted by the diversity of optical depths in the disks, but we show in the following that this ratio presents some characteristic trends that can be explained easily when considering the possible opacity conditions.

The diagrams in Figs. 7a and $\mathrm{b}$ summarize the results displayed in Table D. 1 by plotting the CO integrated emission as a function of the dust emission at the corresponding wavelength, and scaled at $100 \mathrm{pc}$. As some data have no given error bars, or very small ones, we have added a $10 \%$ error bar on the whole sample in order to homogenize the sample and better account for calibration uncertainties. Different colors are used to represent the five categories of disks: blue indicates the CTTS, violet the Herbig AeBe disks, orange the WTTS, red the hybrid disks and green the debris disks. Only 13 sources are common to both diagrams. As for the transitional disks, we do not distinguish them from the CTTS category as their position on the diagrams does not depart from the CTTS group. For the CO $J=2 \rightarrow 1 / 1.3 \mathrm{~mm}$ emission, we have considered 33 sources where both gas and dust are detected. Among them, six sources are displayed with a lower limit arrow, meaning that the $\mathrm{CO}$ emission might be contaminated by the environment. 6 sources have only an upper limit on the gas emission, 3 on the dust emission (one having a lower limit on the gas), and 20 sources have upper limits both on the gas and the dust emission (mostly WTTS from Hardy et al. 2015). For the $\mathrm{CO} J=3 \rightarrow 2 / 0.8 \mathrm{~mm}$ emission, 42 sources have the continuum and gas emission detected, 3 have only upper 


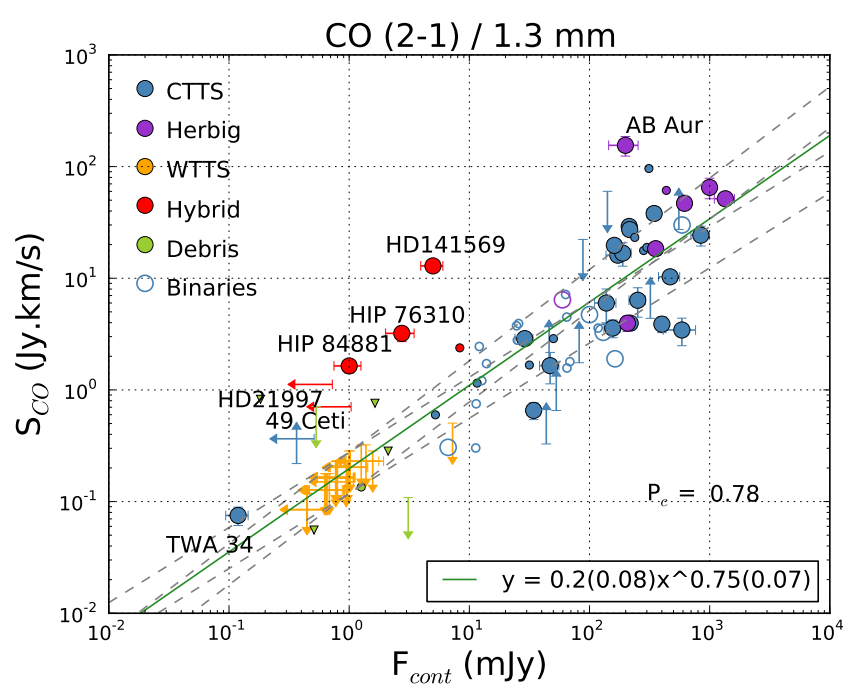

(a)

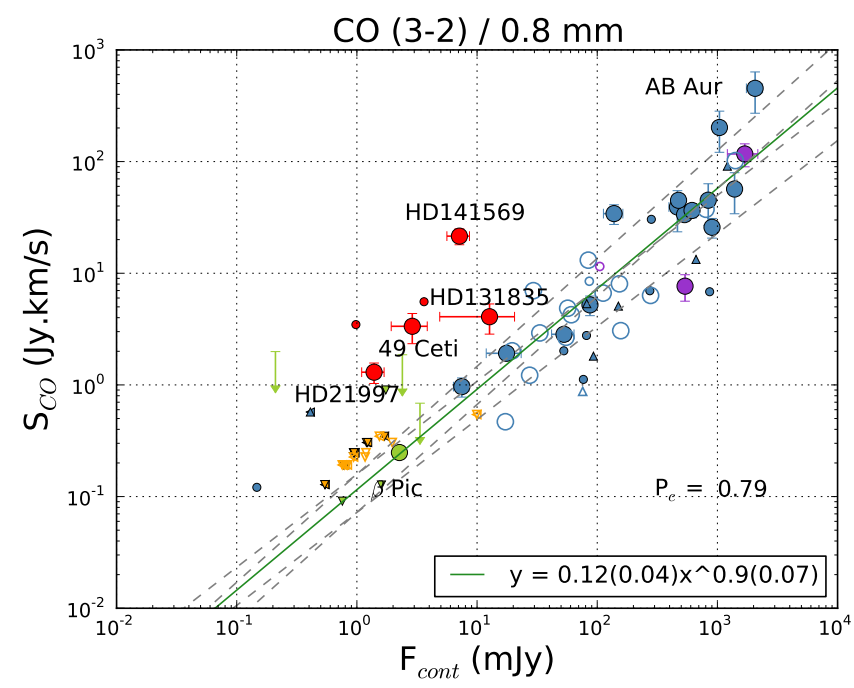

(b)

Fig. 7. a) $\mathrm{CO} J=2 \rightarrow 1$ emission plotted with the corresponding continuum measurement, the fluxes being normalized at $100 \mathrm{pc}$. Blue corresponds to CTTS, violet to Herbig, orange to WTTS, red to hybrid and green to debris disks. The open circles mark the binary systems. The Pearson coefficient of correlation of the data, $P_{c}$, is indicated in the lower right-hand corner. b) As for Fig. 7 a but for the CO J = $3 \rightarrow 2 / 0.8 \mathrm{~mm}$ emission.

limits on the gas emission and 1 has upper limits on the gas and dust emissions.

There is a clear correlation between the emission of the gas and that of dust at $\mathrm{mm}$ wavelengths, with naturally decreasing flux densities for more evolved systems, such as in WTTS or debris disks. The correlation is seen both at $1.3 \mathrm{~mm}$ and $0.8 \mathrm{~mm}$.

The sample spans at least three orders of magnitude in flux density and we observe an almost linear correlation between dust and gas emissions in Figs. 7a and b, with no clear evidence for a slope break (although there are many more sources in the upper right-hand corner of the diagram than in the faint dust/CO emission region where the optically thin regime is expected to be found). There is no obvious difference between CTTS, HAeBe, single, and wide binary stars. A few sources lie well above the global trend: most of them are suspected hybrid disks (HIP 84881, 49 Ceti, HD 21997 and the most prominent outlier HD 141569) with the exception of the Herbig Ae (envelope embedded) star AB Aur. The dispersion around the bestfit line is less than one order of magnitude. The absence of a break in the correlation suggests that the majority of the detected gas disks might still be in the optically thick regime, while optically thin gaseous disks (expected at the last stages of the protoplanetary disk dispersal or in young debris disks) remain mostly undetected so far.

Finally, we notice in Fig. 4 that there could also be a correlation between the $\mathrm{CO}$ integrated flux (scaled at $100 \mathrm{pc}$ ) and the dust fractional luminosity for hybrid disks. However, this trend is based on very few detected gas-rich debris disks so far, and their fractional luminosity spans over approximately one decade only. If it were confirmed, this trend combined with the sensitivity limits of current surveys (at the level of 49 Ceti emission) could partly explain the very low occurrence of CO detections in disks with weak IR excess $\left(L_{\mathrm{IR}} / L_{\star}<5 \times 10^{-4}\right)$. We note that the new candidate HD 23642 does not fit well on that possible trend, although its IR excess is still poorly constrained at far-IR wavelengths. The binary nature of the source (with a larger stellar mass and a suggested larger disk than other hybrid disks) may also be responsible for the discrepancies.

\subsection{Search for correlations between the CO/dust flux ratio and the stellar parameters}

The equivalent width, that is, the ratio $S_{\mathrm{CO}} / F_{\text {cont }}$, has been calculated for the whole sample of 103 sources. We obtain an estimate of $S_{\mathrm{CO}} / F_{\text {cont }}$ for 73 different sources; 46 at $1.3 \mathrm{~mm}$ and 44 at $0.8 \mathrm{~mm}$. The ratio was not calculated when only upper limits exist for both the gas and the continuum. For some sources, the flux was interpolated at 1.3 or $0.8 \mathrm{~mm}$ based on the observed correlations (see Appendix B). The ratio $S_{\mathrm{CO}} / F_{\text {cont }}$ was then plotted against the spectral type (Fig. 8), the stellar mass (Fig. 9), the stellar luminosity (Fig. 10), the accretion rate (Fig. 11), and the age (Fig. 12) of the stars when they are known. We have retrieved the spectral type for 98 sources, the stellar mass for 85 sources and the stellar luminosity for 71 sources.

In the figures, the median value, the first and third quartiles, Q1 and Q3 (respectively delimiting values below which 25\% and $75 \%$ of the sources lie), and the 5th and 95th percentiles, P5 and P95 (respectively delimiting values below which $5 \%$ and $95 \%$ of the sources lie) are drawn for a better understanding. The name of the sources is specified only for values out of P5 and P95.

Spectral type, $M_{\star}$ and $L_{\star}$ : there is no correlation between the ratio $S_{\mathrm{CO}} / F_{\text {cont }}$ and either the spectral type (Fig. 8), stellar mass (Fig. 9), or stellar luminosity (Fig. 10). However, A stars (heavier than $2 M_{\odot}$ ) tend to have a higher $S_{\mathrm{CO}} / F_{\text {cont }}$ ratio. This trend is mainly dominated by the hybrid disks, where the ratio is approximately $1000 \mathrm{~km} \mathrm{~s}^{-1}$, compared to the median value $\sim 100 \mathrm{~km} \mathrm{~s}^{-1}$. Among the Herbig AeBe, only AB Aur shows a high ratio, most likely because of the presence of its envelope (see Sect. 3.7.1).

Accretion rate: we have found in the literature the accretion rates for 40 sources. We do not observe any correlation in the corresponding diagram (Fig. 11). We do not observe any correlations in the diagrams. This may partly reflect the lack of data (only $39 \%$ of the sources have measured accretion rates), in particular for the older population (hybrids and debris). At 


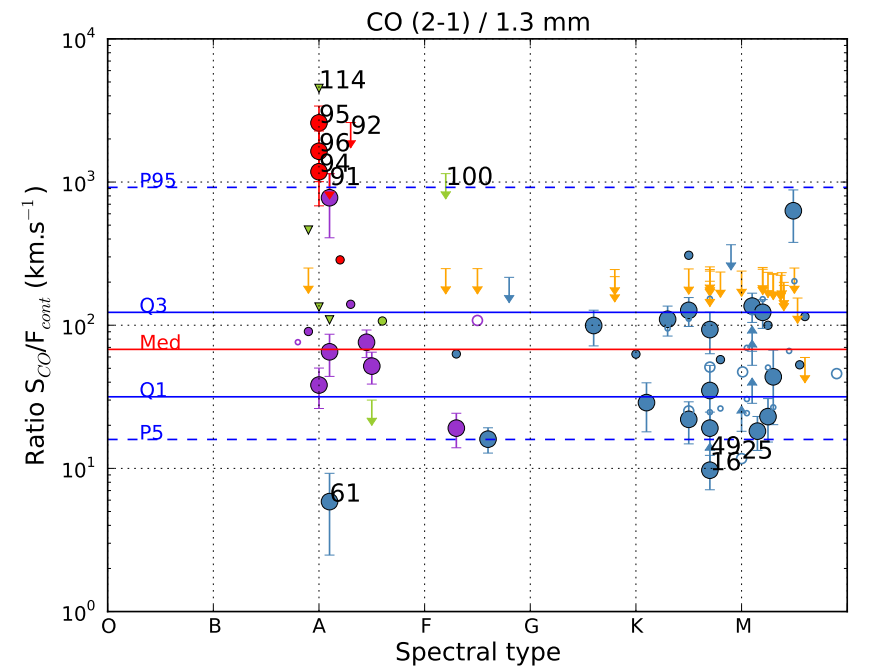

Fig. 8. Ratio $S_{\mathrm{CO}} / F_{\text {cont }}$ plotted against the spectral type of the stars, at $1.3 \mathrm{~mm}$. The color code is the same as in Figs. 7a and b: blue for CTTS, violet for Herbig, orange for WTTS, red for hybrid and green for debris disks. Some statistics are represented by the horizontal lines: the red full line shows the median value of the ratio for the distribution of points, the blue full lines represent the first and third quartiles $(50 \%$ of the points are between these two lines) and the blue dashed lines show the 5th and 95th percentiles ( $90 \%$ of the points between the lines).

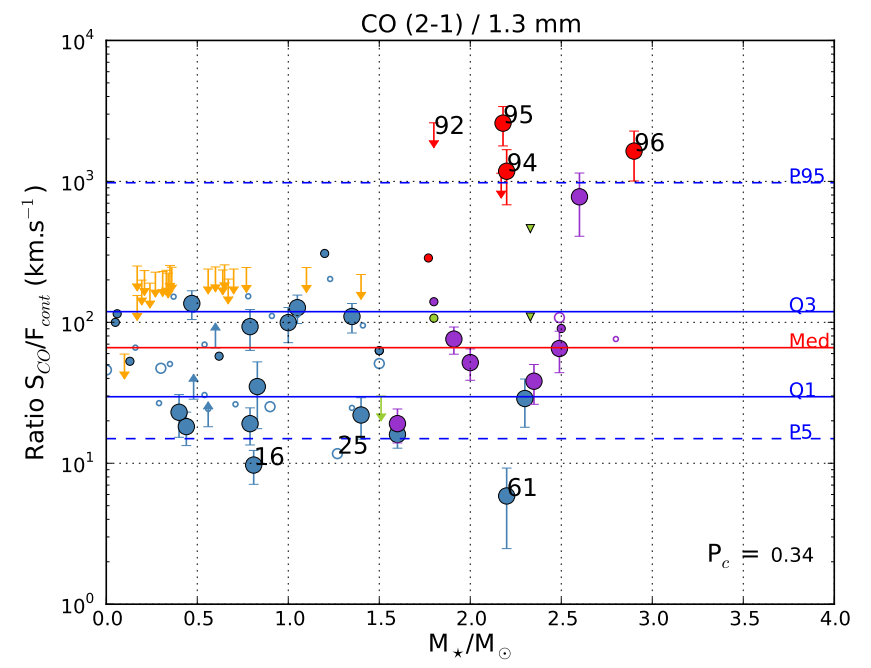

Fig. 9. Ratio $S_{\mathrm{CO}} / F_{\text {cont }}$ at $1.3 \mathrm{~mm}$ plotted against the stellar mass. The color code and lines are the same as in Fig. 8. The Pearson coefficient of correlation of the data, $P_{\mathrm{c}}$, is indicated in the lower right-hand corner.

mm wavelengths, the emission is mostly dominated by the outer parts of the disks, and the sensitivity on inner parts is limited. Since the accretion tracers reflect phenomena occuring close to the star, it is not surprising that the global emission of the disk is not correlated to the local inner accretion signatures. Measurements or at least upper limits on the accretion rates on more evolved stars might help, but it seems that accretion signatures are not a good tracer for evaluating the global evolution of the material around disks.

Age: we have retrieved the age of 82 sources and present the relationship between the ratio $S_{\mathrm{CO}} / F_{\text {cont }}$ and age in Fig. 12 . A trend is observed for older disks to have higher $S_{\mathrm{CO}} / F_{\text {cont }}$ ratios. Nevertheless, as already noted in paragraph 3.6, the trend is

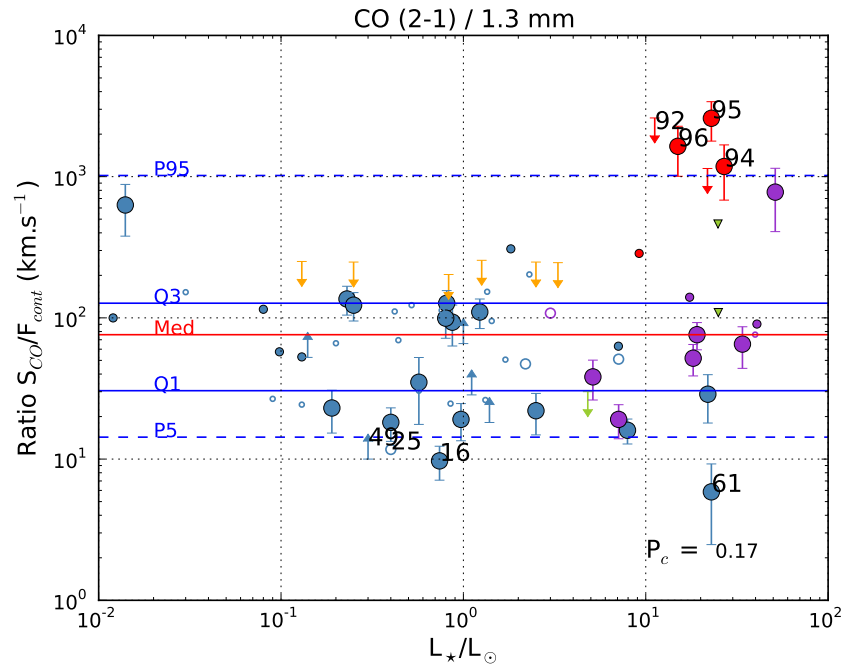

Fig. 10. As Fig. 9 but as a function of stellar luminosity.

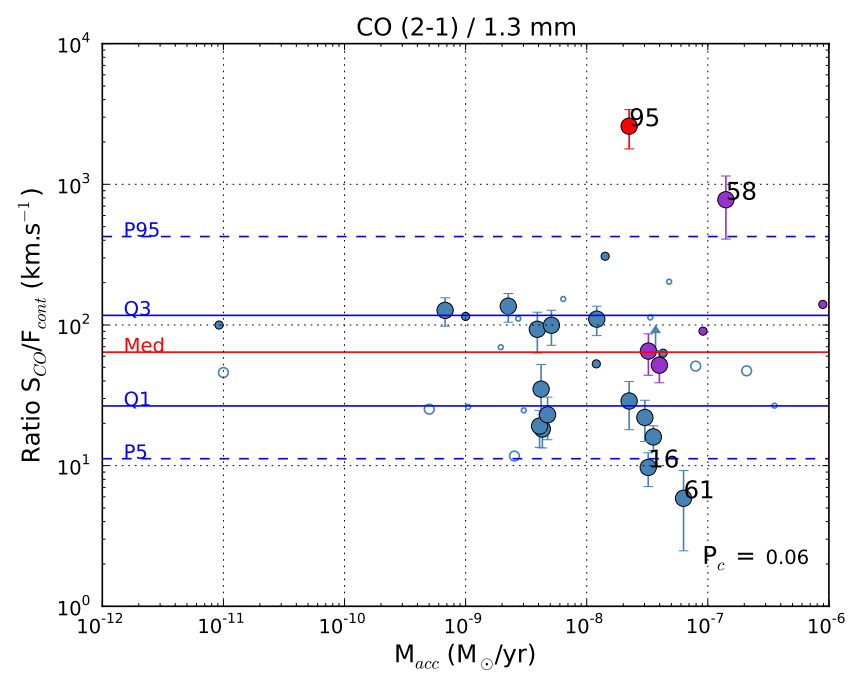

Fig. 11. As Fig. 9 but as a function of accretion rate.

mainly driven by the hybrid disks, and possibly affected by the difficult determination of ages of young stars.

\subsection{Outliers}

Here we list the disks that seem to have an equivalent width $S_{\mathrm{CO}} / F_{\text {cont }}$ departing from the observed median value $100 \mathrm{~km} \mathrm{~s}^{-1}$, that is, lying below the 5th quartile and above the 95th quartile in the different diagrams presented in Sect. 3.6. With the exception of $\mathrm{AB}$ Aur, all sources having a high flux ratio belong to the category of hybrid disks.

\subsubsection{Objects with high equivalent width}

HD 141569. The star of spectral type B9.5V/A0Ve is located $116 \mathrm{pc}$ and has two M-star companions that may be unbound (Reche et al. 2009). The age of the triple system is approximately $5 \pm 3$ Myr (Weinberger et al. 2000; Merín et al. 2004). With a stellar mass of $2 M_{\odot}$, the HD 141569A star+disk system appears in an intermediate evolutionary stage compared to the younger gas-rich system AB Aurigae (Piétu et al. 2005; Tang et al. 2012) and the more evolved $\beta$ Pictoris 


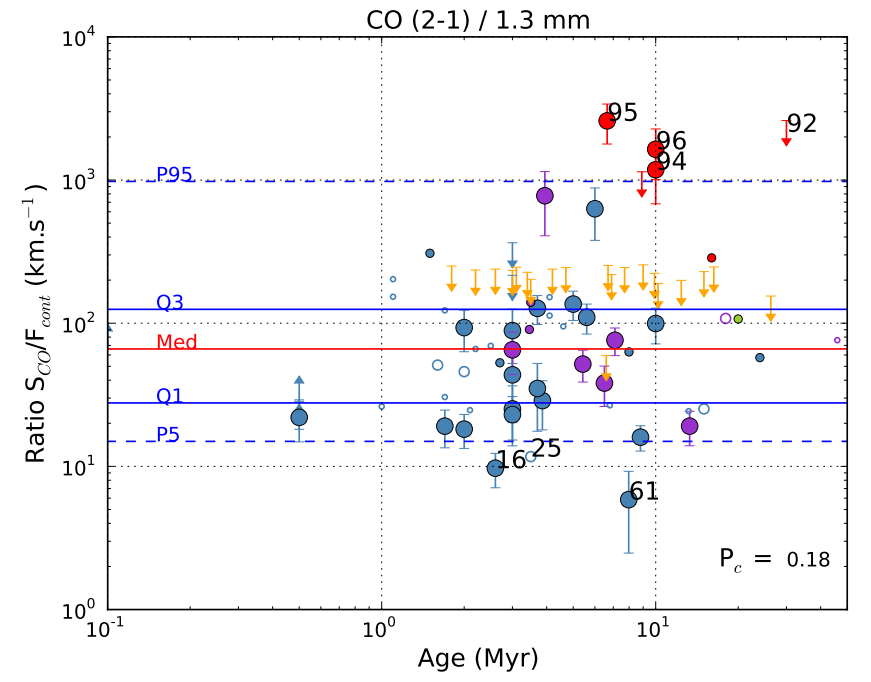

Fig. 12. As Fig. 9 but as a function of system age.

disk. The debris disk of HD 141569 (Meeus et al. 2012, $\left.L_{\mathrm{IR}} / L_{\star}=9 \times 10^{-3}\right)$, presents a complex multiple-ring architecture with spirals and gaps imaged in scattered light by the HST (Augereau et al. 1999; Weinberger et al. 1999; Mouillet et al. 2001; Boccaletti et al. 2003; Clampin et al. 2003). A large quantity of molecular and atomic gas is still present (Zuckerman et al. 1995; Brittain et al. 2003, 2007; Dent et al. 2005; Goto et al. 2006; Salyk et al. 2011; Thi et al. 2014). The CO gas extends out to $\sim 250$ au (Flaherty et al. 2016; Pericaud et al., in prep.).

HD 21997. A gaseous disk around HD 21997 discovered by Moór et al. (2011) in a survey of gas around young debris disks $\left(L_{\mathrm{IR}} / L_{\star}=7.2 \times 10^{-4}\right.$, Chen et al. 2014). Located at $72 \mathrm{pc}$, the A3 star is a member of the Columba association, which is estimated to be 230 Myr old (Moór et al. 2006; Torres et al. 2008). Given the age of the star and the amount of CO, Zuckerman \& Song (2012) have proposed that the gas could be produced by collisions of comets. Nevertheless, the detailed study of the system with ALMA resolved observations (Moór et al. 2013; Kóspál et al. 2013) reveals that the gas and the dust are not co-located, which could imply that they have a different origin: the gas might still be primordial while the dust is of second generation.

49 Ceti. The system is very similar to HD 21997: the spectral type of this $59 \mathrm{pc}$ distant star is A1, and it is associated with the 40 Myr Argus group (Zuckerman \& Song 2012; Torres et al. 2008). It is one of the first debris disks $\left(L_{\mathrm{IR}} / L_{\star}=4.6 \times 10^{-4}\right.$, Chen et al. 2014) where CO gas was detected (Zuckerman et al. 1995). The origin of the gas is still debated, and the comparison of the resolved ALMA observations of the dust with the gas (Hughes et al. 2008) will help to decipher its origin, currently proposed to be either primordial or secondary.

HD 131835. The gas disk around HD 131835 was discovered using the same approach as for HD 21997, during a survey to detect gas in debris disks (Moór et al. 2015). They have detected the $J=3 \rightarrow 2$ transition at a $5 \sigma$ level, but no significant emission is observed for the CO $J=2 \rightarrow 1$ and CO $J=4 \rightarrow 3$. In addition to the gas detection, they have deeply studied the disk thanks to the Herschel, Spitzer, and FEROS instruments.
The dust is resolved at 70 and $100 \mu \mathrm{m}$, extending, on average, to $170 \mathrm{au}$, with a fractional dust luminosity $L_{\mathrm{IR}} / L_{\star}$ of $2.0 \times 10^{-3}$ (Chen et al. 2014). This A2-type star (122 pc) is young (16 Myr, Pecaut et al. 2012), and thus represents another example of a gas-rich debris disk, possibly of the hybrid kind: the analysis of the gas by Moór et al. (2015) does not rule out a primordial origin.

HIP 84881. The disk of HIP 84881 has been revealed by the analysis of WISE photometric data (Rizzuto et al. 2012). Its infrared fractional luminosity is of the same order of magnitude as HD 141569, HD 131835, and $\beta$ Pic $\left(f=3.78^{-3}\right.$, McDonald et al. 2012). This has motivated the search for gas in this young debris disk with ALMA. Both the $\mathrm{CO}$ and continuum are detected at $1.3 \mathrm{~mm}$ (Lieman-Sifry et al. 2016). The dust disk has a marginally resolved inner radius, $<20$ au and extends out to $150 \pm 30$ au.

HIP 76310. In addition to HIP 84881, HIP 76310 is the other new hybrid disk discovered by Lieman-Sifry et al. (2016). The infrared excess has been detected by Spitzer (Carpenter et al. 2006), and is the strongest excess among A-B stars in Upper Sco $\left(L_{\mathrm{IR}} / L_{\star}=9 \times 10^{-4}\right.$ after Chen et al. 2014). The millimeter emission at $1.2 \mathrm{~mm}$ was detected first by Mathews et al. (2012) with the IRAM $30 \mathrm{~m}$, and recently by ALMA, as well as ${ }^{12} \mathrm{CO} J=2 \rightarrow 1$ emission (Lieman-Sifry et al. 2016). The dusty disk has a resolved inner cavity of $\sim 70 \mathrm{au}$.

AB Aurigae. This is a younger Herbig Ae system of the Taurus region, $4 \mathrm{Myr}$ old. The A1-type star is surrounded by a large flared disk of gas and dust. A 100 au inner depletion of material is observed (Piétu et al. 2005), as well as spirals both in the gas and dust warped distributions (Fukagawa et al. 2004; Hashimoto et al. 2011; Tang et al. 2012). A large envelope remains present around the system $(r \sim 1300$ au, Grady et al. 1999), from which material could be accreted despite its evolved age (Tang et al. 2012). Consequently, the high ratio of CO emission compared to the dust might be affected by this remnant envelope.

\subsubsection{Objects with low equivalent width}

HD 245185. This A type star HD 245185 is part or the $\lambda$ Orionis cloud, located $450 \mathrm{pc}$ away, even if the membership of the Taurus region is not excluded (Ansdell et al. 2015). Several studies have demonstrated that the star is accreting material from a primordial disk (Finkenzeller \& Mundt 1984; Herbst \& Shevchenko 1999; de Winter et al. 2001; Hernández et al. 2004, 2010; Acke et al. 2005; Wade et al. 2007; Donehew \& Brittain 2011). Ansdell et al. (2015) have observed the detected CO $J=2 \rightarrow 1$ emission and the dust at $850 \mu \mathrm{m}$. They observe a mid-IR dip in its spectral energy distribution (SED), which could be the sign of a gap in the disk.

DL Tau. A dust disk around DL Tau was first detected by Beckwith et al. (1990), followed by the observation of CO gas (Koerner \& Sargent 1995; Simon et al. 2000), CN (Guilloteau et al. 2014), and $\mathrm{H}_{2} \mathrm{CO}$ (Guilloteau et al. 2013), despite strong confusion. While the $\mathrm{CO}$ and $\mathrm{CN}$ extends out to $\sim 460 \mathrm{au}$, the dust disk is smaller, with an outer radius of 150-180 au (Guilloteau et al. 2011; Kwon et al. 2015). 
Contamination by the molecular cloud may have affected the integrated line flux.

DQ Tau. This is a spectroscopic binary, part of the TaurusAuriga complex, implying a distance of $\sim 140 \mathrm{pc}$ and a young age (1-3 Myr). With an orbital period of 15.8 days, the stellar separation is $\sim 0.1$ au (Mathieu et al. 1997). The authors deduce that the disk truncation effect by the binary is limited to the inner 0.4 au region, and that no effect is visible on the SED compared to a single classical T Tauri disk. Warm CO and dust have been detected in this region $\left(R_{\mathrm{CO}} \sim 0.1 \mathrm{au}\right.$, Carr et al. 2001; $R_{\text {dust }} \sim 0.2$ au, Boden et al. 2009). The system is strongly accreting material from the circumbinary disk, as attested by the variability of the infrared and radio emissions (Salter et al. 2008; Bary \& Petersen 2014). The gas emission is faint, and only ${ }^{12} \mathrm{CO}$ has been detected $\left(\mathrm{CN}\right.$ and $\mathrm{H}_{2} \mathrm{CO}$ search led to non-detections, strong ${ }^{13} \mathrm{CO}$ confusion, Guilloteau et al. 2013). Williams \& Best (2014) estimate the gas disk mass to be lower than $10^{-3} M_{\odot}$ and the dust mass lower than $9 \times 10^{-5} M_{\odot}$.

BP Tau. Like DQ Tau, BP Tau is a member of the Taurus cloud, but is a single star harboring a CO disk extending out to $120 \mathrm{au}$, and a dust disk larger than 100 au (Dutrey et al. 2003). This makes BP Tau one of the most compact disks detected. Dutrey et al. (2003) argue that the ${ }^{12} \mathrm{CO}$ is optically thin, which could explain its low position on the diagram.

\section{Discussion}

\subsection{Interpretation of the $S_{C O} / F_{\text {cont }}$ correlations}

Figures $7 \mathrm{a}$ and $\mathrm{b}$ reveal an interesting correlation that can be interpreted as follows: different regimes of opacity correspond to different regions of the diagrams. Depending on the dust/gas opacity, the emission is governed by different disk physical parameters. Following the standard description of a dust disk (Dutrey et al. 1996), in the optically thin regime, the flux can be written as:

$S_{v}\left(\tau_{v} \ll 1\right)=\frac{2 k T}{D^{2}}\left(\frac{v}{c}\right)^{2} \kappa M$,

where $T$ is the (average) temperature, $\kappa$ the absorption coefficient, and $M$ the mass (each of these parameters being relative to the gas or dust component).

The continuum emission of dust grains is expected to be optically thin at the considered wavelengths even for proto-planetary disks (typically, the optically thick area has a radius of $R \simeq 10$ au at $1.3 \mathrm{~mm}$ for a disk such as that surrounding DM Tau). In contrast, we expect the ${ }^{12} \mathrm{CO}$ gas emission to be mostly in the optically thick regime, where it should then write:

$S_{v}\left(\tau_{v} \gg 1\right) \propto \frac{2 \pi k T}{D^{2}}\left(\frac{v}{c}\right)^{2}\left(R_{\text {out }}^{2}-R_{\text {in }}^{2}\right) \times \cos (i)$,

where $R_{\text {out }}$ and $R_{\text {in }}$ are respectively the outer and inner radii of the disk.

To compare the optically thin emission to the optically thick one, we can develop Eq. (1) and write the mass as a function of the inner and outer disk radii. Indeed, the mass can be derived from the surface density (see Appendix A.2 for details), $\Sigma(r)=\Sigma_{0}\left(\frac{R}{R_{0}}\right)^{-p}:$

$M \propto \frac{2 \pi \Sigma_{0} R_{0}^{2}}{2-p}\left(\frac{R_{\text {out }}}{R_{0}}-\frac{R_{\text {in }}}{R_{0}}\right)^{2-p}$.

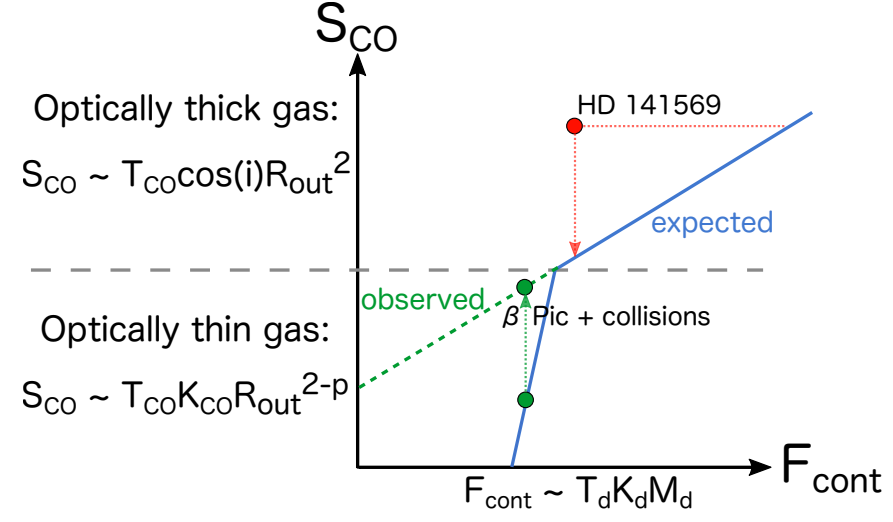

Fig. 13. Diagram showing the physical parameters which dominate the emission of the CO gas and dust (see Sect. 4.2 for details). The higher part of the plot represents the optically thick regime of the gas, and the lower part represents the optically thin regime. In a hybrid disk such as HD 141569, the dust may evolve faster than the gas in a first step, moving the disk out of the correlation. In a second step, the natural gas dissipation moves downwards in position. On the contrary, debris disks are expected to lie in the optically thin region. For $\beta$ Pic, the position of the disk in the diagram could be higher than expected because of the gas enhancement produced by collisional events. For both sources, the arrows show the expected path in the diagram resulting from their possible evolutions.

At first order, we thus see that the emission in the optically thick regime (upper part of the diagrams), neglecting the inner radius, varies as $R_{\text {out }}^{2}$, while the optically thin emission varies as $R_{\text {out }}^{2-p}$. The transition between the optically thick and thin regimes for the gas emission should then result in a slope break, as sketched out in Fig. 13. Others parameters, such as the inclination, the difference between the dust and the gas temperatures, or the presence of a large inner hole may be responsible for the dispersion of the points around the global trend in these diagrams, but should not affect the dependence on $R_{\text {out }}$.

Interestingly, we do not observe a break in the correlations of Figs. 7a and b, all the observed sources show the same slope suggesting optically thick $\mathrm{CO}$ emission all along.

\subsection{A possible diagram of evolution}

In Figs. 7a and b, the systems span a large range of properties (luminosity, mass and age etc.), from envelope-embedded young Class II sources to transition and young debris disks, and a large fraction of the disk evolution sequence is represented. Therefore, we investigate whether or not the correlation observed between the $\mathrm{CO}$ emission and the thermal $\mathrm{mm} / \mathrm{sub}-\mathrm{mm}$ flux can be interpreted as a sequence of evolution in the life of disks.

First of all, it is important to highlight that the detected hybrid objects are either isolated or in loose associations, and may not be representative of disk evolution in dense clusters. Observations show that the fraction of disks seems lower in older clusters (Haisch et al. 2001; Hernández et al. 2007), which indicates that the disk lifetime is approximately 5 Myr. Survival of disks in general, however, might not be as short as the age determined in these studies. Indeed, in clusters, the rich stellar environment may enhance the disk dispersal and lead to shorter lifetime estimates than for isolated stars (Pfalzner et al. 2014; Vincke et al. 2015). Pfalzner et al. (2014) evaluate that at least $30 \%$, and possibly as much as $50 \%$, of the disks are living for more than $10 \mathrm{Myr}$. The (isolated) hybrid disks we are 
discovering nowadays might thus be the first of a long series. The fact that they are found around isolated A-stars contributes to the ease of their detection, since their disks tend to be more massive and luminous.

Nevertheless, as a proto-planetary disk evolves, its surface density becomes eroded under the effects of viscous evolution, photo-evaporation and planet formation. As a consequence, its dust and gas emissions are expected to decrease until they reach the optically thin domain of debris disks where, for a matter of sensitivity, only the continuum emission of (second generation) dust remains detectable in most cases. The accretion rate is also often interpreted as an indicator of the evolution of the disks, since the accretion signatures are supposed to decrease when the gas dissipates. Hartmann et al. (1998) have identified a decrease of the accretion rates with the stellar age, but the study was restricted to CTTS disks. Several studies have also examined the evolution of the accretion rate in transitional disks compared to CTTS ones, since transitional disks are supposed to be in a more evolved stage than CTTS. It appears that the disk fraction showing accretion signatures is significantly lower in the transitional disk population, but when they are present, the accretion level is similar to the CTTS level, or less than an order of magnitude below (Fang et al. 2013; Sicilia-Aguilar et al. 2010; Espaillat et al. 2012; Najita et al. 2007). Figure 11 does not show any correlations.

In Figs. $7 a$ and $b$, there is no significant difference between young (gas rich) disks orbiting Herbig Ae and T Tauri stars. The linear shape of the $\mathrm{CO} /$ grains emission distribution in Figs. $7 \mathrm{a}$ and $b$, and the small dispersion around the mean trend suggest that the gas and dust components generally evolve concurrently, with comparable dissipation timescales, even if in our sample the distribution of CTTS (including binaries, which are all along the main correlation) is more homogenous than that of Herbig Ae stars.

Although the number of faint disks simultaneously detected in CO line and continuum remains too small for a robust analysis in the bottom-left region of the diagram, all hybrid disk candidates (red symbols) with evolved dust and gas of (partially) primordial origin clearly lie above the correlation line. This reveals either a deficit of dust emission or an excess of $\mathrm{CO}$ emission. Similarly, we also observe an enhanced flux ratio in Figs. 8-10 for the hybrid disk population.

\subsection{Origin of hybrid disks}

Herbig Ae stars may follow a different pathway, that is, on a specific trend lying above that of low-mass stars or with a different slope in Figs. 7a and b, but the lack of Herbig stars in the range $F_{\mathrm{CO}}=10-500 \mathrm{mJy}$ prevents us from drawing conclusions. However, in this region of the diagram, sensitivity is not an issue and the apparent absence of Herbig Ae stars in the vicinity of the hybrid disks group might be better explained by an effect of disk dissipation rather than by a detection bias.

Two scenarios, corresponding to different evolution pathways in Figs. 7a and b, may be invoked.

\subsubsection{Excess of $\mathrm{CO}$ by comet/planetesimal destruction}

In this first case, dramatic events such as analogs of the Late Heavy Bombardment in our solar system (Gomes et al. 2005) or a massive collision may be invoked to make faint sources move towards the upper part of the diagram. Such a scenario is suspected for $\beta$ Pictoris, whose large $\mathrm{CO}$ intensity is thought to originate from a recent collision between Mars-size objects or in an enhanced collision rate due to planetesimals trapped in mean-motion resonances with an unseen planet (Dent et al. 2014). This suggests that its current location in our diagram, on the same line as optically thick disks, is transient (see Fig. 13), while the "steady-state" regime of the icy debris collisional cascade may produce insufficient $\mathrm{CO}$ gas for most debris disks to be detectable (see recent ALMA non-detections of $\mathrm{CO}$ around the nearby debris disks Fomalhaut and HD 107146, Matrà et al. 2015; Ricci et al. 2015).

For HD 21997, a typical hybrid disk, Kóspál et al. (2013) have evaluated the $\mathrm{CO}$ mass from the $\mathrm{C}^{18} \mathrm{O}$ emission. They constrain it to $4-8 \times 10^{-2} M_{\oplus}$, that is, $\sim 4 \times 10^{23} \mathrm{~kg}$ and $\sim 7 \times 10^{20} \mathrm{~kg}$ for ${ }^{12} \mathrm{CO}$ and $\mathrm{C}^{18} \mathrm{O}$ (assuming an isotopic ratio of 560), respectively. They estimated that such a $\mathrm{CO}$ mass cannot be purely produced by cometary collisions, as at least 6000 Hale-Bopp like comets would need to be totally destroyed each year.

To evaluate the relevance of a comet/planetesimal destruction scenario in explaining the observed CO mass, we outline another approach. We use the following assumptions:

- $M_{\text {disk }}=0.1 M_{\star}=0.2 M_{\odot}$ for Herbig stars;

- $M_{\text {disk }} \approx M_{\mathrm{H}_{2}}$

- most of the $\mathrm{O}$ is in the form of $\mathrm{H}_{2} \mathrm{O}$; the $\mathrm{H}_{2} \mathrm{O}$ content of the disk is then given by cosmic oxygen abundance;

- the $\mathrm{CO}$ content is derived using a $\left[\mathrm{CO} / \mathrm{H}_{2} \mathrm{O}\right]$ molecular abundance ratio in comets/planetesimals of $10 \%$. This value is found for CO-rich comets such as Hale-Bopp and Lovejoy in the solar system; the median being $4 \%$ (see e.g., Paganini et al. 2014; Mumma \& Charnley 2011);

- $10 \%$ of this CO content ends up locked in comets/ planetesimals;

- $X 18$, the ${ }^{12} \mathrm{CO} / \mathrm{C}^{18} \mathrm{O}$ ratio in gas and in comets is similar; we use $X 18=560$.

These assumptions give an estimate of the initial mass of ${ }^{12} \mathrm{CO}$ and $\mathrm{C}^{18} \mathrm{O}$ in the disk:

$M_{\mathrm{CO}}^{\mathrm{init}}<M_{\mathrm{disk}} \times m_{\mathrm{O} / \mathrm{H}} \times\left[\frac{\mathrm{CO}}{\mathrm{H}_{2} \mathrm{O}}\right] \times \frac{m_{\mathrm{CO}}}{m_{\mathrm{O}}}$,

where $m_{\mathrm{CO}}$ is the $\mathrm{CO}$ molecular mass, $m_{\mathrm{O}}$ the oxygen atomic mass, and $m_{\mathrm{O} / \mathrm{H}}$ the fraction mass of oxygen compared to $\mathrm{H}$. Taking the cosmic abundance $[\mathrm{O} / \mathrm{H}]=3.4 \times 10^{-4}$ yields $m_{\mathrm{O} / \mathrm{H}}=$ $16 \times[\mathrm{O} / \mathrm{H}] \approx 0.005$. The numerical application gives $M_{\mathrm{CO}}^{\text {init }}<$ $4 \times 10^{26} \mathrm{~kg}$. The mass ultimately locked in comets/planetesimals is thus $M_{\mathrm{CO}}^{\text {lock }}<4 \times 10^{25} \mathrm{~kg}$ and $7 \times 10^{22} \mathrm{~kg}$ for ${ }^{12} \mathrm{CO}$ and $\mathrm{C}^{18} \mathrm{O}$, respectively.

From the observed CO column density in HD 21997, neglecting the shielding from dust grains which have a low column density and taking into account both the stellar UV flux and the ISM flux, Kóspál et al. (2013) found that the lifetime would be $<30000 \mathrm{yr}$ for ${ }^{12} \mathrm{CO}$ and $<6000 \mathrm{yr}$ for $\mathrm{C}^{18} \mathrm{O}$. The observed $\mathrm{C}^{18} \mathrm{O}$ content would thus need to be replenished at a rate of $10^{17} \mathrm{~kg} \mathrm{yr}^{-1}$. The available reservoir of comets estimated above would then be exhausted in less than $6 \times 10^{5} \mathrm{yr}$. Without a reservoir, the remaining ${ }^{12} \mathrm{CO}$ would only last $<30000 \mathrm{yr}$ longer.

This should be compared to the required duration of the phenomenon: $3 \mathrm{Myr}$ given the detection rate (approximately 7\%) and age of the oldest system (approximately $40 \mathrm{Myr}$ ). Thus, in spite of the uncertainties in our assumptions (particularly in our conservative estimate of the $\mathrm{CO}$ production rate), it is very unlikely that the scenario of comets/planetesimals destruction could explain the whole $\mathrm{CO}$ emission observed in hybrid disks. 


\subsubsection{Deficit of $\mathrm{mm}$ emission - grain growth}

In the second scenario, the observed correlation suggests that disks share common properties during most of their lifetime, differing in characteristic size and surface density, but not in gas to dust ratio, and evolve along the correlation line because of a global decrease in gas and dust content. Hybrid disks would appear when the dust properties change significantly enough to affect the $\mathrm{CO}$ flux to $\mathrm{mm}$ flux ratio. A potential cause is grain growth, as observed in the inner regions of disks (Guilloteau et al. 2011; Pérez et al. 2012), which lowers the dust opacity and thus the mm flux without affecting the gas. A further reduction of the NIR to UV dust opacity can allow the onset of photo-evaporation, which will then reduce the gas content faster than the dust content (Gorti et al. 2015). In this scenario, the small number of hybrid disks is in accordance with the short duration of the gas-rich, optically thin dust phase.

Unfortunately, the gas to dust ratio is difficult to estimate from the observations, which only prove a high $\mathrm{CO}$ to continuum flux ratio in the hybrid disk phase. The more detailed studies of HD 21997 (Kóspál et al. 2013) and HD 141569 (Di Folco et al., in prep.) indicate gas to dust mass ratios much larger than the canonical ISM value of 100 in the outer disk regions $(R>100 \mathrm{au})$ sampled by the observations. However, the paucity of resolved hybrid disks precludes any firm conclusion on the gas to dust mass ratio, and thus on the validity of the proposed scenario. We note however that a fast dust evolution in these disks around A-type stars is also supported by the observational finding from NIR excess that the (small) dust dispersal timescale was found to be shorter around intermediate-mass stars than around low-mass stars (e.g., Hillenbrand et al. 1993; Kennedy \& Kenyon 2009; Ribas et al. 2015).

Current searches have focussed on A stars: establishing the fraction of hybrid disks around lower-mass stars would be an essential step in indentifying the cause of their existence. This requires much more sensitive searches, only possible with ALMA (see Lieman-Sifry et al. 2016). Spatially resolved studies such as those of HD 21997 and HD 141569 are also needed to move from a global $\mathrm{CO}$ to dust flux ratio to a view of their respective distributions, and to guide us towards the understanding of this elusive phase.

Acknowledgements. This research has made use of the SIMBAD database, operated at CDS, Strasbourg, France. This publication is based on observations carried out with the IRAM 30-m telescope and the Atacama Pathfinder Experiment (APEX). IRAM is supported by INSU/CNRS (France), MPG (Germany) and IGN (Spain). APEX is a collaboration between the MaxPlanck-Institut fur Radioastronomie, the European Southern Observatory, and the Onsala Space Observatory. This paper makes use of the following ALMA data: ADS/JAO.ALMA\#2011.0.00150.S， ADS/JAO.ALMA\#2013.1.00163.S and ADS/JAO.ALMA\#2011.0.00780.S. ALMA is a partnership of ESO (representing its member states), NSF (USA) and NINS (Japan), together with NRC (Canada), NSC and ASIAA (Taiwan), and KASI (Republic of Korea), in cooperation with the Republic of Chile. The Joint ALMA Observatory is operated by ESO, AUI/NRAO and NAOJ. The paper is also based on observations collected at the European Organisation for Astronomical Research in the Southern Hemisphere under ESO programmes 094.C-0161(A).095.C-0742(A) This work was supported by "Programme National de Physique Stellaire" (PNPS) and "Programme National de Planétologie" (PNP) from INSU/CNRS Finally, we warmly acknowledge the anonymous referee for her/his detailed comments which helped to improve the paper.

\section{References}

Abt, H. A., \& Levato, H. 1978, PASP, 90, 201

Acke, B., van den Ancker, M. E., \& Dullemond, C. P. 2005, A\&A, 436, 209

Akeson, R. L., \& Jensen, E. L. N. 2014, ApJ, 784, 62

Andrews, S. M., \& Williams, J. P. 2005, ApJ, 631, 1134

Andrews, S. M., \& Williams, J. P. 2007, ApJ, 659, 705
Andrews, S. M., Wilner, D. J., Hughes, A. M., Qi, C., \& Dullemond, C. P. 2009 ApJ, 700, 1502

Andrews, S. M., Wilner, D. J., Hughes, A. M., Qi, C., \& Dullemond, C. P. 2010, ApJ, 723, 1241

Andrews, S. M., Rosenfeld, K. A., Kraus, A. L., \& Wilner, D. J. 2013, ApJ, 771, 129

Ansdell, M., Williams, J. P., \& Cieza, L. A. 2015, ApJ, 806, 221

Augereau, J. C., Lagrange, A. M., Mouillet, D., \& Ménard, F. 1999, A\&A, 350, L51

Banzatti, A., Testi, L., Isella, A., et al. 2011, A\&A, 525, A12

Bary, J. S., \& Petersen, M. S. 2014, ApJ, 792, 64

Bast, J. E., Brown, J. M., Herczeg, G. J., van Dishoeck, E. F., \& Pontoppidan, K. M. 2011, A\&A, 527, A119

Beckwith, S. V. W., Sargent, A. I., Chini, R. S., \& Guesten, R. 1990, AJ, 99, 924 Beskrovnaya, N. G., Pogodin, M. A., Miroshnichenko, A. S., et al. 1999, A\&A, 343,163

Binks, A. S., \& Jeffries, R. D. 2014, MNRAS, 438, L11

Boccaletti, A., Augereau, J.-C., Marchis, F., \& Hahn, J. 2003, ApJ, 585, 494

Boden, A. F., Akeson, R. L., Sargent, A. I., et al. 2009, ApJ, 696, L111

Booth, M., Kennedy, G., Sibthorpe, B., et al. 2013, MNRAS, 428, 1263

Bowler, B. P., Liu, M. C., Kraus, A. L., \& Mann, A. W. 2014, ApJ, 784, 65

Breger, M. 1987, ApJ, 319, 754

Brittain, S. D., Rettig, T. W., Simon, T., et al. 2003, ApJ, 588, 535

Brittain, S. D., Simon, T., Najita, J. R., \& Rettig, T. W. 2007, ApJ, 659, 685

Brown, J. M., Blake, G. A., Qi, C., et al. 2009, ApJ, 704, 496

Caceres, C., Hardy, A., Schreiber, M. R., et al. 2015, ApJ, 806, L22

Canovas, H., Caceres, C., Schreiber, M. R., et al. 2016, MNRAS, 458, L29

Carpenter, J. M., Mamajek, E. E., Hillenbrand, L. A., \& Meyer, M. R. 2006, ApJ, 651, L49

Carr, J. S., Mathieu, R. D., \& Najita, J. R. 2001, ApJ, 551, 454

Castellani, V., Degl'Innocenti, S., Prada Moroni, P. G., \& Tordiglione, V. 2002, MNRAS, 334, 193

Chapillon, E., Guilloteau, S., Dutrey, A., \& Piétu, V. 2008, A\&A, 488, 565

Chapillon, E., Guilloteau, S., Dutrey, A., Piétu, V., \& Guélin, M. 2012, A\&A, 537, A60

Chen, C. H., Mittal, T., Kuchner, M., et al. 2014, ApJS, 211, 25

Chen, C. H., Sargent, B. A., Bohac, C., et al. 2006, ApJS, 166, 351

Cieza, L. A., Schreiber, M. R., Romero, G. A., et al. 2012, ApJ, 750, 157

Clampin, M., Krist, J. E., Ardila, D. R., et al. 2003, AJ, 126, 385

Czekala, I., Andrews, S. M., Jensen, E. L. N., et al. 2015, ApJ, 806, 154

Czekala, I., Andrews, S. M., Torres, G., et al. 2016, ApJ, 818, 156

de Winter, D., van den Ancker, M. E., Maira, A., et al. 2001, A\&A, 380, 609

Dent, W. R. F., Greaves, J. S., \& Coulson, I. M. 2005, MNRAS, 359, 663

Dent, W. R. F., Thi, W. F., Kamp, I., et al. 2013, PASP, 125, 477

Dent, W. R. F., Wyatt, M. C., Roberge, A., et al. 2014, Science, 343, 1490

Donehew, B., \& Brittain, S. 2011, AJ, 141, 46

Duchêne, G., Monin, J.-L., Bouvier, J., \& Ménard, F. 1999, A\&A, 351, 954

Dutrey, A., Guilloteau, S., Duvert, G., et al. 1996, A\&A, 309, 493

Dutrey, A., Guilloteau, S., \& Simon, M. 2003, A\&A, 402, 1003

Dutrey, A., Wakelam, V., Boehler, Y., et al. 2011, A\&A, 535, A104

Duvert, G., Guilloteau, S., Ménard, F., Simon, M., \& Dutrey, A. 2000, A\&A, 355,165

Eiroa, C., Marshall, J. P., Mora, A., et al. 2013, A\&A, 555, A11

Espaillat, C., D'Alessio, P., Hernández, J., et al. 2010, ApJ, 717, 441

Espaillat, C., Ingleby, L., Hernández, J., et al. 2012, ApJ, 747, 103

Fairlamb, J. R., Oudmaijer, R. D., Mendigutía, I., Ilee, J. D., \& van den Ancker,

M. E. 2015, MNRAS, 453, 976

Fang, M., van Boekel, R., Bouwman, J., et al. 2013, A\&A, 549, A15

Finkenzeller, U., \& Mundt, R. 1984, A\&AS, 55, 109

Flaherty, K. M., Hughes, A. M., Andrews, S. M., et al. 2016, ApJ, 818, 97

Fukagawa, M., Hayashi, M., Tamura, M., et al. 2004, ApJ, 605, L53

Garcia Lopez, R., Natta, A., Testi, L., \& Habart, E. 2006, A\&A, 459, 837

Garufi, A., Quanz, S. P., Avenhaus, H., et al. 2013, A\&A, 560, A105

Gomes, R., Levison, H. F., Tsiganis, K., \& Morbidelli, A. 2005, Nature, 435, 466

Gorti, U., Hollenbach, D., \& Dullemond, C. P. 2015, ApJ, 804, 29

Goto, M., Usuda, T., Dullemond, C. P., et al. 2006, ApJ, 652, 758

Grady, C. A., Woodgate, B., Bruhweiler, F. C., et al. 1999, ApJ, 523, L151

Groenewegen, M. A. T., Decin, L., Salaris, M., \& De Cat, P. 2007, A\&A, 463, 579

Grosso, N., Alves, J., Wood, K., et al. 2003, ApJ, 586, 296

Guilloteau, S., Dutrey, A., Piétu, V., \& Boehler, Y. 2011, A\&A, 529, A105

Guilloteau, S., Di Folco, E., Dutrey, A., et al. 2013, A\&A, 549, A92

Guilloteau, S., Simon, M., Piétu, V., et al. 2014, A\&A, 567, A117

Haisch, Jr., K. E., Lada, E. A., \& Lada, C. J. 2001, ApJ, 553, L153

Hales, A. S., De Gregorio-Monsalvo, I., Montesinos, B., et al. 2014, AJ, 148, 47

Hardy, A., Caceres, C., Schreiber, M. R., et al. 2015, A\&A, 583, A66

Hartigan, P., \& Kenyon, S. J. 2003, ApJ, 583, 334

Hartigan, P., Strom, K. M., \& Strom, S. E. 1994, ApJ, 427, 961 
Hartigan, P., Edwards, S., \& Ghandour, L. 1995, ApJ, 452, 736 Hartmann, L., Calvet, N., Gullbring, E., \& D’Alessio, P. 1998, ApJ, 495, 385 Hashimoto, J., Tamura, M., Muto, T., et al. 2011, ApJ, 729, L17

Herbig, G. H., \& Bell, K. R. 1988, in Third Catalog of Emission-Line Stars of the Orion Population: 3 (Santa Cruz: Lick Observatory), 90

Herbst, W., \& Shevchenko, V. S. 1999, AJ, 118, 1043

Herczeg, G. J., \& Hillenbrand, L. A. 2014, ApJ, 786, 97

Hernández, J., Calvet, N., Briceño, C., Hartmann, L., \& Berlind, P. 2004, AJ, 127,1682

Hernández, J., Calvet, N., Hartmann, L., et al. 2005, AJ, 129, 856

Hernández, J., Hartmann, L., Megeath, T., et al. 2007, ApJ, 662, 1067

Hernández, J., Morales-Calderon, M., Calvet, N., et al. 2010, ApJ, 722, 1226

Hillenbrand, L. A., Massey, P., Strom, S. E., \& Merrill, K. M. 1993, AJ, 106, 1906

Howard, C. D., Sandell, G., Vacca, W. D., et al. 2013, ApJ, 776, 21

Hughes, A. M., Wilner, D. J., Kamp, I., \& Hogerheijde, M. R. 2008, ApJ, 681, 626

Isella, A., Testi, L., Natta, A., et al. 2007, A\&A, 469, 213

Isella, A., Carpenter, J. M., \& Sargent, A. I. 2009, ApJ, 701, 260

Isella, A., Natta, A., Wilner, D., Carpenter, J. M., \& Testi, L. 2010, ApJ, 725 1735

Jensen, E. L. N., Mathieu, R. D., \& Fuller, G. A. 1996, ApJ, 458, 312

Kastner, J. H., Zuckerman, B., Hily-Blant, P., \& Forveille, T. 2008, A\&A, 492

Kastner, J. H., Hily-Blant, P., Sacco, G. G., Forveille, T., \& Zuckerman, B. 2010, ApJ, 723, L248

Kennedy, G. M., \& Kenyon, S. J. 2009, ApJ, 695, 1210

Kennedy, G. M., Murphy, S. J., Lisse, C. M., et al. 2014, MNRAS, 438, 3299

Koerner, D. W., \& Sargent, A. I. 1995, AJ, 109, 2138

Kóspál, Á., Moór, A., Juhász, A., et al. 2013, ApJ, 776, 77

Kraus, A. L., Ireland, M. J., Cieza, L. A., et al. 2014, ApJ, 781, 20

Kwon, W., Looney, L. W., Mundy, L. G., \& Welch, W. J. 2015, ApJ, 808, 102

Lieman-Sifry, J., Hughes, A. M., Carpenter, J. M., et al. 2016, ApJ, 828, 25

Luhman, K. L., Allen, P. R., Espaillat, C., Hartmann, L., \& Calvet, N. 2010, ApJS, 186, 111

MacGregor, M. A., Wilner, D. J., Andrews, S. M., \& Hughes, A. M. 2015, ApJ, 801,59

Mannings, V., \& Emerson, J. P. 1994, MNRAS, 267, 361

Manoj, P., Bhatt, H. C., Maheswar, G., \& Muneer, S. 2006, ApJ, 653, 657

Mathews, G. S., Williams, J. P., Ménard, F., et al. 2012, ApJ, 745, 23

Mathieu, R. D., Stassun, K., Basri, G., et al. 1997, AJ, 113, 1841

Matrà, L., Panić, O., Wyatt, M. C., \& Dent, W. R. F. 2015, MNRAS, 447, 3936

Matthews, B., Kennedy, G., Sibthorpe, B., et al. 2014, ApJ, 780, 97

Mazoyer, J., Boccaletti, A., Augereau, J.-C., et al. 2014, A\&A, 569, A29

McDonald, I., Zijlstra, A. A., \& Boyer, M. L. 2012, MNRAS, 427, 343

Meeus, G., Montesinos, B., Mendigutía, I., et al. 2012, A\&A, 544, A78

Mendigutía, I., Calvet, N., Montesinos, B., et al. 2011, A\&A, 535, A99

Merín, B., Montesinos, B., Eiroa, C., et al. 2004, A\&A, 419, 301

Montesinos, B., Eiroa, C., Mora, A., \& Merín, B. 2009, A\&A, 495, 901

Moór, A., Ábrahám, P., Derekas, A., et al. 2006, ApJ, 644, 525

Moór, A., Ábrahám, P., Juhász, A., et al. 2011, ApJ, 740, L7

Moór, A., Juhász, A., Kóspál, Á., et al. 2013, ApJ, 777, L25

Moór, A., Henning, T., Juhász, A., et al. 2015, ApJ, 814, 42

Mora, A., Merín, B., Solano, E., et al 2001, A\&A, 378, 116

Morales, F. Y., Werner, M. W., Bryden, G., et al. 2009, ApJ, 699, 1067

Mouillet, D., Lagrange, A. M., Augereau, J. C., \& Ménard, F. 2001, A\&A, 372, L61

Mumma, M. J., \& Charnley, S. B. 2011, ARA\&A, 49, 471

Munari, U., Dallaporta, S., Siviero, A., et al. 2004, A\&A, 418, L31

Najita, J. R., Strom, S. E., \& Muzerolle, J. 2007, MNRAS, 378, 369

Natta, A., Testi, L., Muzerolle, J., et al. 2004a, A\&A, 424, 603

Natta, A., Testi, L., Neri, R., Shepherd, D. S., \& Wilner, D. J. 2004b, A\&A, 416, 179

Natta, A., Testi, L., \& Randich, S. 2006, A\&A, 452, 245

Nilsson, R., Liseau, R., Brandeker, A., et al. 2010, A\&A, 518, A40

Öberg, K. I., Qi, C., Fogel, J. K. J., et al. 2010, ApJ, 720, 480

Owen, J. E., \& Clarke, C. J. 2012, MNRAS, 426, L96

Paganini, L., Mumma, M. J., Villanueva, G. L., et al. 2014, ApJ, 791, 122

Panić, O., van Dishoeck, E. F., Hogerheijde, M. R., et al. 2010, A\&A, 519, A110

Panić, O., Holland, W. S., Wyatt, M. C., et al. 2013, MNRAS, 435, 1037

Pascucci, I., Gorti, U., Hollenbach, D., et al. 2006, ApJ, 651, 1177

Patel, R. I., Metchev, S. A., \& Heinze, A. 2014, ApJS, 212, 10

Pecaut, M. J., Mamajek, E. E., \& Bubar, E. J. 2012, ApJ, 746, 154

Pérez, L. M., Carpenter, J. M., Chandler, C. J., et al. 2012, ApJ, 760, L17
Perez, S., Casassus, S., Ménard, F., et al. 2015, ApJ, 798, 85

Pfalzner, S., Steinhausen, M., \& Menten, K. 2014, ApJ, 793, L34

Piétu, V., Dutrey, A., \& Kahane, C. 2003, A\&A, 398, 565

Piétu, V., Guilloteau, S., \& Dutrey, A. 2005, A\&A, 443, 945

Piétu, V., Dutrey, A., Guilloteau, S., Chapillon, E., \& Pety, J. 2006, A\&A, 460, L43

Piétu, V., Dutrey, A., \& Guilloteau, S. 2007, A\&A, 467, 163

Raman, A., Lisanti, M., Wilner, D. J., Qi, C., \& Hogerheijde, M. 2006, AJ, 131, 2290

Reboussin, L., Guilloteau, S., Simon, M., et al. 2015, A\&A, 578, A31

Reche, R., Beust, H., \& Augereau, J.-C. 2009, A\&A, 493, 661

Rhee, J. H., Song, I., Zuckerman, B., \& McElwain, M. 2007, ApJ, 660, 1556

Ribas, Á., Bouy, H., \& Merín, B. 2015, A\&A, 576, A52

Ricci, L., Testi, L., Natta, A., et al. 2010, A\&A, 512, A15

Ricci, L., Testi, L., Natta, A., Scholz, A., \& de Gregorio-Monsalvo, I. 2012, ApJ, 761, L20

Ricci, L., Testi, L., Natta, A., et al. 2014, ApJ, 791, 20

Ricci, L., Carpenter, J. M., Fu, B., et al. 2015, ApJ, 798, 124

Rigliaco, E., Pascucci, I., Duchene, G., et al. 2015, ApJ, 801, 31

Riviere-Marichalar, P., Barrado, D., Montesinos, B., et al. 2014, A\&A, 565, A68

Rizzuto, A. C., Ireland, M. J., \& Zucker, D. B. 2012, MNRAS, 421, L97

Roberge, A., \& Weinberger, A. J. 2008, ApJ, 676, 509

Roberge, A., Kamp, I., Montesinos, B., et al. 2013, ApJ, 771, 69

Rodriguez, D. R., van der Plas, G., Kastner, J. H., et al. 2015, A\&A, 582, L5

Romero, G. A., Schreiber, M. R., Cieza, L. A., et al. 2012, ApJ, 749, 79

Rosenfeld, K. A., Andrews, S. M., Hughes, A. M., Wilner, D. J., \& Qi, C. 2013, ApJ, 774, 16

Salter, D. M., Hogerheijde, M. R., \& Blake, G. A. 2008, A\&A, 492, L21

Salyk, C., Blake, G. A., Boogert, A. C. A., \& Brown, J. M. 2011, ApJ, 743, 112

Salyk, C., Herczeg, G. J., Brown, J. M., et al. 2013, ApJ, 769, 21

Salyk, C., Pontoppidan, K., Corder, S., et al. 2014, ApJ, 792, 68

Schneider, A., Song, I., Melis, C., Zuckerman, B., \& Bessell, M. 2012, ApJ, 757, 163

Schneider, A., Song, I., Melis, C., et al. 2013, ApJ, 777, 78

Scoville, N. Z., Sargent, A. I., Sanders, D. B., et al. 1986, ApJ, 303, 416

Sheret, I., Dent, W. R. F., \& Wyatt, M. C. 2004, MNRAS, 348, 1282

Sicilia-Aguilar, A., Henning, T., \& Hartmann, L. W. 2010, ApJ, 710, 597

Simon, M., Dutrey, A., \& Guilloteau, S. 2000, ApJ, 545, 1034

Smith, R., Churcher, L. J., Wyatt, M. C., Moerchen, M. M., \& Telesco, C. M. 2009, A\&A, 493, 299

Stauffer, J. R., Schultz, G., \& Kirkpatrick, J. D. 1998, ApJ, 499, L199

Su, K. Y. L., Rieke, G. H., Stansberry, J. A., et al. 2006, ApJ, 653, 675

Sylvester, R. J., Dunkin, S. K., \& Barlow, M. J. 2001, MNRAS, 327, 133

Tang, Y.-W., Guilloteau, S., Piétu, V., et al. 2012, A\&A, 547, A84

Thi, W. F., van Dishoeck, E. F., Blake, G. A., et al. 2001, ApJ, 561, 1074

Thi, W.-F., Pinte, C., Pantin, E., et al. 2014, A\&A, 561, A50

Torres, G. 2003, IBVS, 5402

Torres, C. A. O., Quast, G. R., da Silva, L., et al. 2006, A\&A, 460, 695

Torres, C. A. O., Quast, G. R., Melo, C. H. F., \& Sterzik, M. F. 2008, in Handbook of Star Forming Regions, Vol. II, ed. B. Reipurth, 757

Tsukagoshi, T., Momose, M., Hashimoto, J., et al. 2014, ApJ, 783, 90

Vacca, W. D., \& Sandell, G. 2011, ApJ, 732, 8

van Boekel, R., Min, M., Waters, L. B. F. M., et al. 2005, A\&A, 437, 189

Vincke, K., Breslau, A., \& Pfalzner, S. 2015, A\&A, 577, A115

Wade, G. A., Bagnulo, S., Drouin, D., Landstreet, J. D., \& Monin, D. 2007, MNRAS, 376, 1145

Walsh, C., Juhász, A., Pinilla, P., et al. 2014, ApJ, 791, L6

Weinberger, A. J., Becklin, E. E., Schneider, G., et al. 1999, ApJ, 525, L53

Weinberger, A. J., Rich, R. M., Becklin, E. E., Zuckerman, B., \& Matthews, K. 2000, ApJ, 544, 937

Welsh, B. Y., \& Montgomery, S. 2013, PASP, 125, 759

White, R. E. 2003, ApJS, 148, 487

White, R. J., \& Ghez, A. M. 2001, ApJ, 556, 265

White, R. J., Ghez, A. M., Reid, I. N., \& Schultz, G. 1999, ApJ, 520, 811

Wilking, B. A., Meyer, M. R., Robinson, J. G., \& Greene, T. P. 2005, AJ, 130, 1733

Williams, J. P., \& Andrews, S. M. 2006, ApJ, 653, 1480

Williams, J. P., \& Best, W. M. J. 2014, ApJ, 788, 59

Williams, J. P., Cieza, L. A., Andrews, S. M., et al. 2013, MNRAS, 435, 1671

Wyatt, M. C., Smith, R., Su, K. Y. L., et al. 2007, ApJ, 663, 365

Zhang, K., Isella, A., Carpenter, J. M., \& Blake, G. A. 2014, ApJ, 791, 42

Zuckerman, B., \& Song, I. 2004, ApJ, 603, 738

Zuckerman, B., \& Song, I. 2012, ApJ, 758, 77

Zuckerman, B., Forveille, T., \& Kastner, J. H. 1995, Nature, 373, 494 


\section{Appendix A: Mass calculations}

\section{A.1. From integrated flux $S_{C O}$}

From Scoville et al. (1986), the mean column density averaged over the beam writes:

$N=\frac{k}{h v} \frac{3 k}{8 \pi^{3} B \mu^{2}} \frac{T_{x}+\frac{h B}{3 k}}{(J+1) \cdot \exp \left(-\frac{h v}{k T_{x}}\right)} \frac{\tau}{1-\mathrm{e}^{-\tau}} \int T_{\mathrm{B}} \mathrm{d} v$,

where $B$ is the rotational constant of the observed molecule, $\mu$ its permanent dipole moment, $J$ the rotational number of the lower state of the transition, $T_{x}$ is the excitation temperature, $\tau$ the optical depth and $T_{\mathrm{B}}$ the brigntness temperature. This expression assumes the excitation temperature is high compared to the background temperature (see Scoville et al. 1986 for details). The total $\mathrm{H}_{2}$ mass is related to this column density thanks to the mean atomic weight of the gas $\mu_{\mathrm{G}}$, the dihydrogen mass $m_{\mathrm{H}_{2}}$, the abundance ratio of $\mathrm{CO}$ to $\mathrm{H}_{2} X$, the distance of the source $\mathrm{D}$ and the beam solid angle $\Omega_{\mathrm{S}}$ :

$M_{\mathrm{H}_{2}}=N \frac{\mu_{\mathrm{G}} m_{\mathrm{H}_{2}}}{X\left(\mathrm{CO} / \mathrm{H}_{2}\right)} D^{2} \Omega_{\mathrm{S}}$.

Using the Planck's law, we thus have

$M_{\mathrm{H}_{2}}=\frac{k}{h v} \frac{3 c D^{2} \mu_{\mathrm{G}} m_{\mathrm{H}_{2}}}{16 v^{2} \pi^{3} B \mu^{2} X} \frac{T_{x}+\frac{h B}{3 k}}{(J+1) \cdot \exp \left(-\frac{h v}{k T_{x}}\right)} \frac{\tau}{1-\mathrm{e}^{-\tau}} \cdot 10^{-23} S_{\mathrm{CO}}$,

where all terms are in S.I. units, except the integrated flux $S_{\mathrm{CO}}$ which should be expressed in Jy $\mathrm{km} \mathrm{s}^{-1}$. We can sum up by

$M_{\mathrm{H}_{2}}\left(M_{\oplus}\right)=K_{1} \times 10^{-11} \frac{D^{2}(\mathrm{pc})}{X} \frac{T_{x}+\frac{h B}{3 k}}{\exp \left(-K_{2} / T_{x}\right)} \frac{\tau}{1-\mathrm{e}^{-\tau}} S_{\mathrm{CO}}$

For the CO molecule, $B=1.93 \mathrm{~cm}^{-1}$, that is, $5.79 \times 10^{10} \mathrm{~Hz}$, $\mu=0.1098$ debye, that is, $\mu^{2}=1.2 \times 10^{-51}$ S.I., and $\mu_{\mathrm{G}}=1.36$, which implies for the $J=2 \rightarrow 1$ transition: $K_{1}=4.83, K_{2}=11.1$ and for the $J=3 \rightarrow 2$ transition: $K_{1}=0.953$ and $K_{2}=16.6$. We have taken the usual value of $10^{-4}$ for $X$, the abundance of $\mathrm{CO}$ to $\mathrm{H}_{2}$. In this paper we have set the values of the excitation temperature and opacity to $T_{\mathrm{ex}}=40 \mathrm{~K}$ and $\tau=1$.

\section{A.2. From DiskFit modeling}

Error bars on $\Sigma_{0}$ determined by the code DiskFit can be used to determine the $\mathrm{H}_{2}$ mass as follows:

$\Sigma_{\mathrm{m}}(r)=\Sigma_{0}\left(\frac{r}{r_{0}}\right)^{-p}\left(\mathrm{~cm}^{-2}\right)$

$N_{\mathrm{CO}}=\int_{r_{\text {int }}}^{r_{\text {out }}} \int_{0}^{2 \pi} \Sigma_{\mathrm{m}}(r) r \mathrm{~d} r \mathrm{~d} \theta$

$N_{\mathrm{CO}}=\frac{2 \pi \Sigma_{0} r_{0}^{2}}{2-p}\left({\frac{r_{\mathrm{out}}}{r_{0}}}^{2-p}-{\frac{r_{\mathrm{out}}}{r_{0}}}^{2-p}\right)$,

$M_{\mathrm{CO}}=N_{\mathrm{CO}} m_{\mathrm{CO}}$,

where $\Sigma_{\mathrm{m}}$ is the molecular surface density, $\Sigma_{0}$ its value at radius $r_{0}, r_{\text {out }}$ the outer radius of the disk, $N_{\mathrm{CO}}$ the number of $\mathrm{CO}$ molecules in the disk, $m_{\mathrm{CO}}$ the molecular mass of the $\mathrm{CO}$, and $M_{\mathrm{CO}}$ the $\mathrm{CO}$ mass in the disk $(\mathrm{kg})$. To retrieve the $\mathrm{H}_{2}$ mass: $X=N_{\mathrm{CO}} / N_{\mathrm{H}_{2}}=1 \times 10^{-4}$, then $N_{\mathrm{H}_{2}}=N_{\mathrm{CO}} / X$ and $M_{\mathrm{H}_{2}}=N_{\mathrm{H}_{2}} m_{\mathrm{H}_{2}}$ where $N_{\mathrm{H}_{2}}$ is the number of molecule of $\mathrm{H}_{2}, m_{\mathrm{H}_{2}}$ its molecular mass and $M_{\mathrm{H}_{2}}$ the total mass of $\mathrm{H}_{2}$.

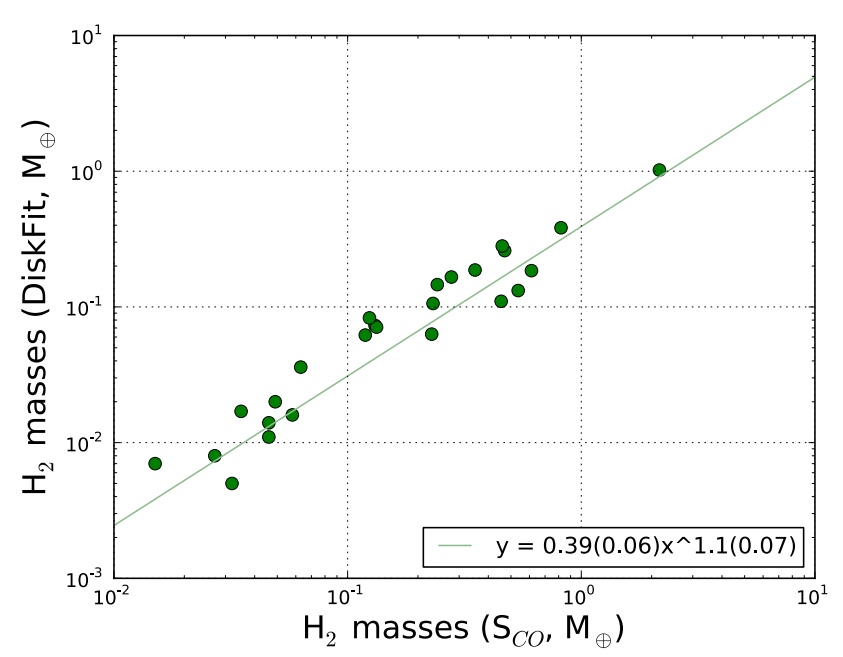

Fig. A.1. Masses of $\mathrm{H}_{2}$ determined for the debris disks observed with APEX/IRAM with the DiskFit method on $Y$ axis and with formula (A.4) on $X$ axis.

\section{A.3. Comparison of the two methods}

Figure A.1 presents the masses determined by the two methods. The methods give consistent results within a factor three. The points showing strong deviations are HD 2772, HD 159082 (below the line), and HD 23642 (above the line).

\section{Appendix B: Interpolation of $\mathrm{CO}$ and dust emission}

Figures B.1 $\mathrm{a}$ and $\mathrm{b}$ show the correlation between the observations of the continuum and gas emission at the two wavelengths from the literature. A linear regression of the data gives the two following equations, that we have used to fill in the missing values (see Sect. 2.2). When no error is given in the literature, we have assumed a $10 \%$ error.

$S_{\mathrm{CO}(3-2)}=1.7^{ \pm 0.4} \times\left(S_{\mathrm{CO}(2-1)}\right)^{1.05^{ \pm 0.05}}$,

$F_{\text {cont }}^{0.8 \mathrm{~mm}}=1.3^{ \pm 0.5} \times\left(F_{\mathrm{cont}}^{1.3 \mathrm{~mm}}\right)^{1.09^{ \pm 0.06}}$.

Figure B. 2 shows the correlation between the $\mathrm{CO}$ and dust emission, without interpolating the missing values. Only detections of CTTS and Herbig are used to calculate the linear regression. When the error on a measurement was unknown, it was fixed to $20 \%$ of the value. A $10 \%$ uncertainty was added to those given in Table D. 1 for the linear regression.

Table B.1. Ratio of fluxes and derived $\mathrm{H}_{2}$ masses for sources of the debris disks surveys where $\mathrm{CO}$ is detected.

\begin{tabular}{lcc}
\hline \hline Source & $\mathrm{CO}(3-2) / \mathrm{CO}(2-1)$ & $M_{\mathrm{H}_{2}}^{32} / M_{\mathrm{H}_{2}}^{21}$ \\
\hline 49 Ceti & 4.75 & 1.08 \\
HD 141569 & 1.68 & 0.38 \\
HD 21997 & 1.16 & 0.26 \\
HD 131835 & 1.71 & 0.78 \\
\hline
\end{tabular}


J. Péricaud et al.: The hybrid disks: a search and study to better understand evolution of disks

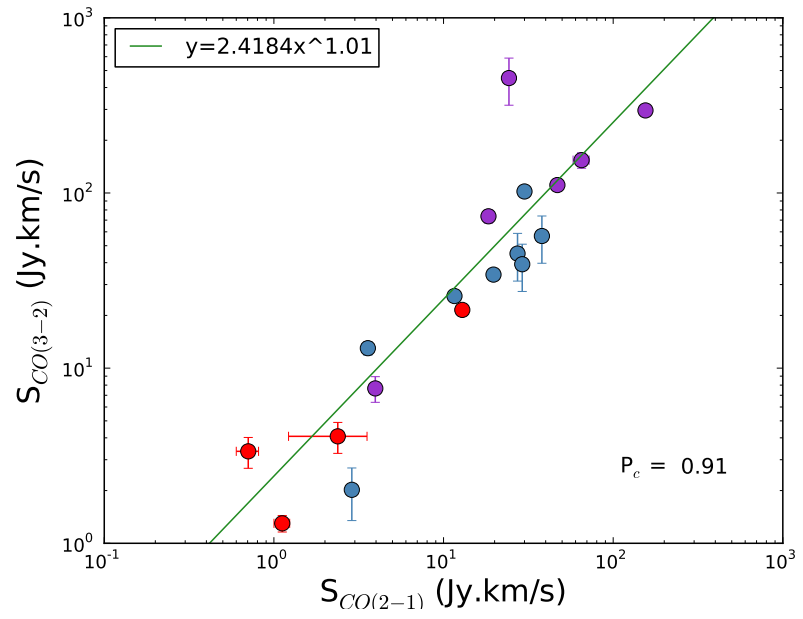

(a)

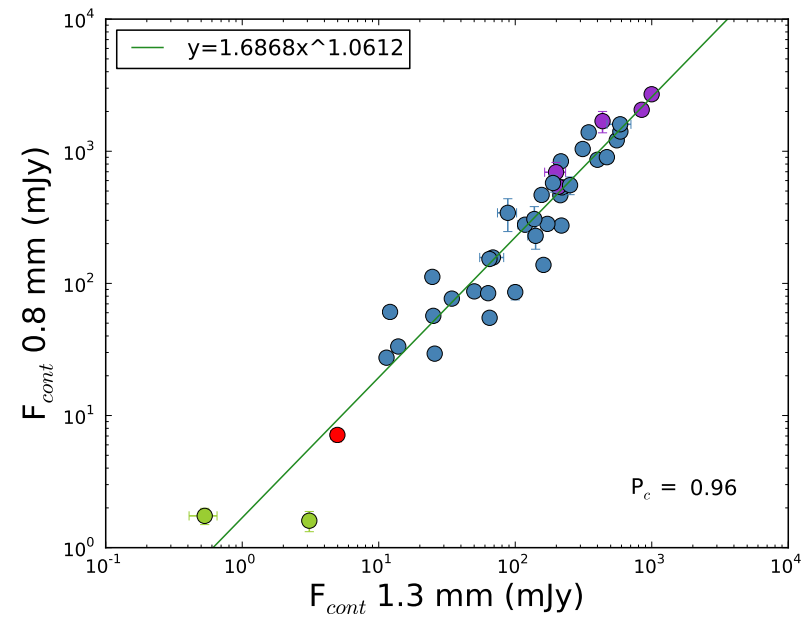

(b)

Fig. B.1. a) Emission of the CO $J=3 \rightarrow 2$ plotted against the CO $J=2 \rightarrow 1$ emission. The Pearson coefficient of correlation of the data, $P_{\mathrm{c}}$, is indicated in the lower right-hand corner. b) Emission of the continuum at $0.8 \mathrm{~mm}$ plotted against the $1.3 \mathrm{~mm}$ continuum emission. The Pearson coefficient of correlation of the data, $P_{\mathrm{c}}$, is indicated in the lower right-hand corner. Fluxes are rescaled at 100 pc. Same symbols as for Fig. B.2.

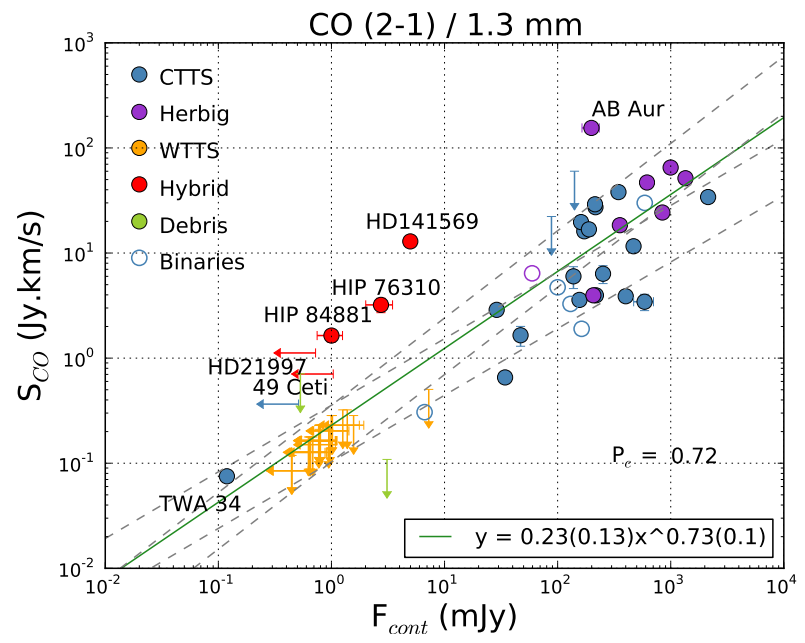

(a)

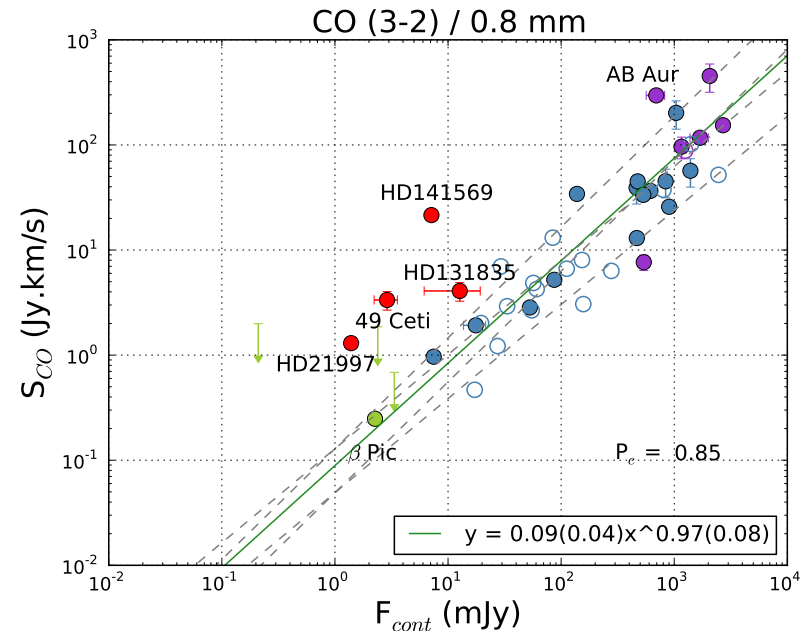

(b)

Fig. B.2. a) Emission of the $\mathrm{CO} J=2 \rightarrow 1$ plotted against the $1.3 \mathrm{~mm}$ continuum emission. The Pearson coefficient of correlation of the data, $P_{\mathrm{c}}$, is indicated in the lower right-hand corner. No extrapolated data are used here, only observations are reported and used to do the linear regression (green full line, equation indicated in the lower right-hand corner). b) Same as a), for the CO $J=3 \rightarrow 2$ emission against the $0.8 \mathrm{~mm}$ continuum emission. Fluxes are rescaled at 100 pc. 


\section{Appendix C: Correlations with stellar parameters}

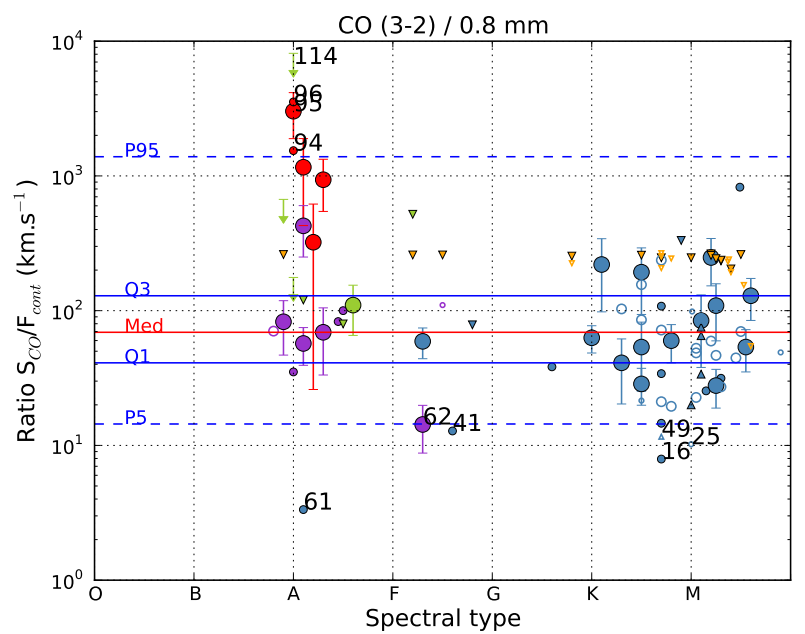

Fig. C.1. Ratio $S_{\mathrm{CO}} / F_{\text {cont }}$ plotted against the spectral types of the stars, at $0.8 \mathrm{~mm}$. The color code is the same as in Figs. 7a and b: blue for CTTS, violet for Herbig, orange for WTTS, red for hybrid and green for debris disks. Some statistics is represented by the lines: the red full line shows the median value of the ratio for the distribution of points, the blue full lines represent the first and third quartiles (50\% of the points are between these two lines) and the blue dashed lines shows the 5th and 95th percentiles (90\% of the points between the lines).
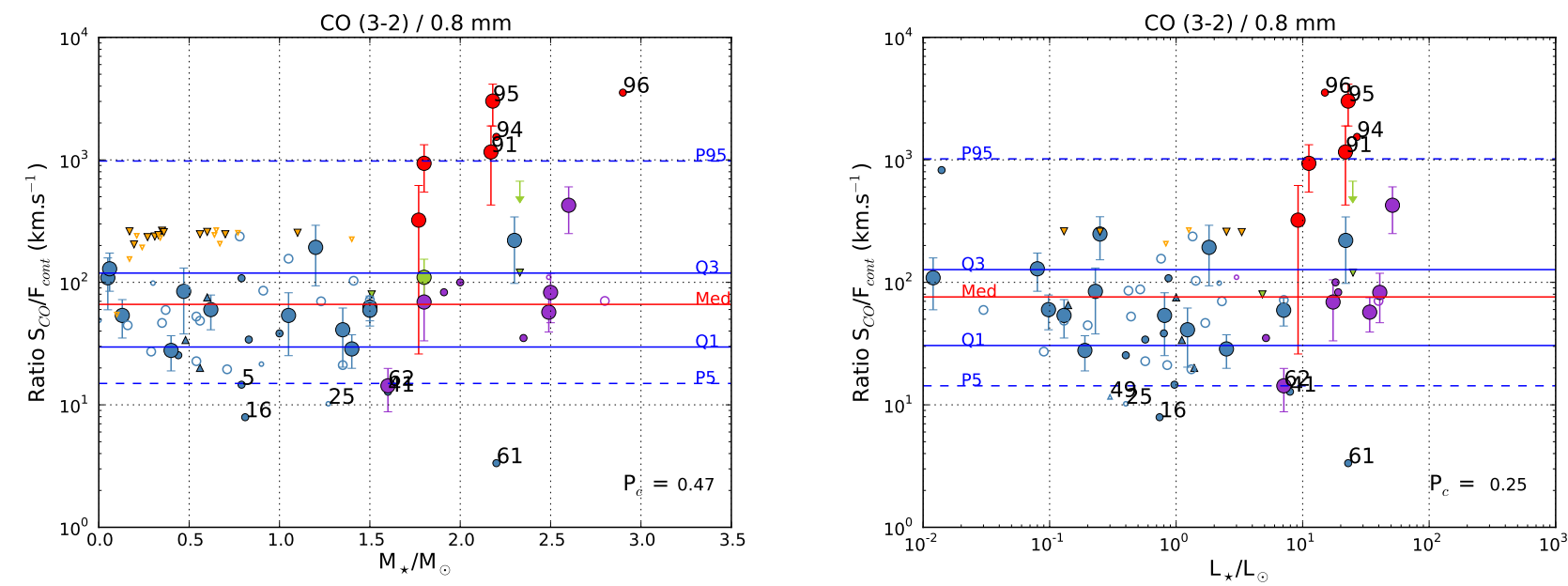

Fig. C.2. Left: ratio $S_{\mathrm{CO}} / F_{\text {cont }}$ plotted against the stellar mass, at $0.8 \mathrm{~mm}$. The color code and lines are the same as in Fig. 9. The Pearson coefficient of correlation of the data, $P_{\mathrm{c}}$, is indicated in the lower right-hand corner. Right: ratio $S_{\mathrm{Co}} / F_{\text {cont }}$ plotted against the stellar luminosity, at $0.8 \mathrm{~mm}$. The color code and lines are the same as in Fig. 10. The Pearson coefficient of correlation of the data, $P_{\mathrm{c}}$, is indicated in the lower right-hand corner. 
J. Péricaud et al.: The hybrid disks: a search and study to better understand evolution of disks
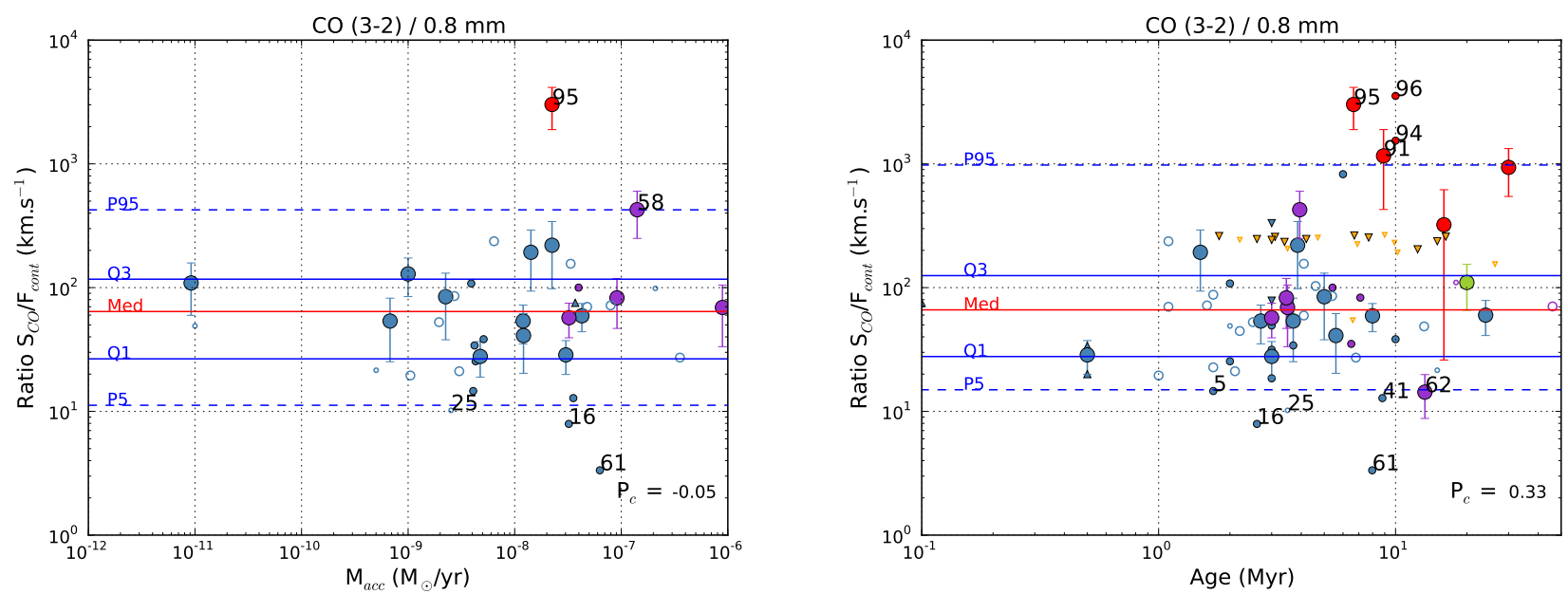

Fig. C.3. Left: ratio $S_{\mathrm{CO}} / F_{\text {cont }}$ plotted against the accretion rate, at $0.8 \mathrm{~mm}$. The color code and lines are the same as in Fig. 11 . The Pearson coefficient of correlation of the data, $P_{\mathrm{c}}$, is indicated in the lower right-hand corner. Right: ratio $S_{C O} / F_{\text {cont }}$ plotted against the age of the system, at $0.8 \mathrm{~mm}$. The color code and lines are the same as in Fig. 12. The Pearson coefficient of correlation of the data, $P_{\mathrm{c}}$, is indicated in the lower right-hand corner. 


\section{Appendix D: Additional tables}

Table D.1. ${ }^{12} \mathrm{CO}$ and continuum emission used to plot Figs. $7 \mathrm{a}$ and $\mathrm{b}$.

\begin{tabular}{|c|c|c|c|c|c|c|}
\hline ID & Name & $\begin{array}{l}\mathrm{CO} J=2 \rightarrow 1 \\
\quad\left(\mathrm{Jy} \mathrm{km} \mathrm{s}^{-1}\right)\end{array}$ & $\begin{array}{l}\text { Continuum } \\
1.3 \mathrm{~mm}(\mathrm{mJy})\end{array}$ & $\begin{array}{l}\mathrm{CO} J=3 \rightarrow 2 \\
\left(\mathrm{Jy} \mathrm{km} \mathrm{s}^{-1}\right)\end{array}$ & $\begin{array}{c}\text { Continuum } \\
0.8 \mathrm{~mm}(\mathrm{mJy})\end{array}$ & References \\
\hline 1 & CIDA 1 & $0.9 \pm 0.8$ & $16 \pm 8$ & $1.45 \pm 0.05$ & $27 \pm 3$ & 1 \\
\hline 2 & IRAS $04113+2758$ & $10 \pm 12$ & $196 \pm 96$ & 19.1 & $410 \pm 5$ & 2 \\
\hline 3 & CY Tau & $2.02 \pm 0.08$ & $111 \pm 3$ & $3.6 \pm 1.0$ & $140 \pm 5$ & $3,4,5$ \\
\hline 4 & ${ }^{\dagger}$ FQ Tau & $0.2 \pm 0.2$ & $6 \pm 3$ & 0.239 & $8.8 \pm 1.2$ & 2 \\
\hline 5 & BP Tau & $1.11 \pm 0.08$ & $58.2 \pm 1.3$ & $1.90 \pm 0.59$ & $130 \pm 7$ & $3,4,5$ \\
\hline 6 & RY Tau & 6.6 & $229 \pm 17$ & $123 \pm 37$ & $560 \pm 30$ & $6,7,8,5$ \\
\hline 7 & ${ }^{\dagger} \mathrm{FV}$ Tau A & $1.3 \pm 1.5$ & $6.17 \pm 0.16$ & 2.17 & $31 \pm 1.4$ & 9,2 \\
\hline 8 & ${ }^{\dagger} \mathrm{FW}$ Tau c & 0.156 & $3.4 \pm 0.2$ & $0.2 \pm 0.1$ & $4.9 \pm 2.4$ & 51 \\
\hline 9 & ${ }^{\dagger}$ FX Tau & $0.9 \pm 1.0$ & $7.1 \pm 0.15$ & 1.49 & $17 \pm 0.9$ & 9,2 \\
\hline 10 & ${ }^{\dagger} \mathrm{DK}$ Tau & $0.9 \pm 1.1$ & $35 \pm 7$ & 1.56 & $66 \pm 1.0$ & 9,2 \\
\hline 11 & ${ }^{\dagger}$ HK Tau A & $2.3 \pm 2.7$ & $33 \pm 0.15$ & 4.1 & $78 \pm 1.1$ & 9,2 \\
\hline 12 & ${ }^{\dagger}$ HK Tau B & $1.9 \pm 2.2$ & $12.6 \pm 0.15$ & 3.39 & $57 \pm 1.3$ & 9,2 \\
\hline 13 & V710 Tau & $1.8 \pm 2.1$ & $59.18 \pm 0.33$ & 3.23 & $142 \pm 1.5$ & 9,2 \\
\hline 14 & GG Tau & $13 \pm 16$ & $546 \pm 298$ & $53 \pm 15$ & $1255 \pm 57$ & 8,5 \\
\hline 15 & GK Tau A & $2.00 \pm 2.33$ & $5.33 \pm 0.56$ & 3.56 & $15 \pm 1$ & 9,2 \\
\hline 16 & DL Tau & $1.98 \pm 0.12$ & $204 \pm 2$ & $3.5 \pm 1.1$ & $440 \pm 40$ & $3,4,10$ \\
\hline 17 & ${ }^{\dagger} \mathrm{HN}$ Tau & $1.4 \pm 1.7$ & $12.8 \pm 0.2$ & 2.48 & $37 \pm 1.1$ & 9,2 \\
\hline 18 & DM Tau & $14.87 \pm 0.12$ & $109 \pm 2.4$ & $20 \pm 6$ & $237 \pm 12$ & $11,4,8,5$ \\
\hline 19 & ${ }^{\dagger}$ IT Tau B & $0.6 \pm 0.7$ & $6.5 \pm 3.1$ & 1.03 & $10 \pm 0.9$ & 2 \\
\hline 20 & AA Tau & $8.2 \pm 0.16$ & $88 \pm 9$ & $15.5 \pm 4.7$ & $144 \pm 5$ & 11,5 \\
\hline 21 & ${ }^{\dagger} \mathrm{HO}$ Tau & $0.8 \pm 0.9$ & $17.06 \pm 0.27$ & 1.36 & $38 \pm 1$ & 9,2 \\
\hline 22 & ${ }^{\dagger} \mathrm{HBC} 411 \mathrm{~B}$ & $0.4 \pm 0.4$ & $5.8 \pm 0.27$ & 0.624 & $14 \pm 1$ & 9,2 \\
\hline 23 & LkCa 15 & $13.94 \pm 0.15$ & $110 \pm 2$ & $23 \pm 7$ & $428 \pm 11$ & $11,4,8,5$ \\
\hline 24 & $\mathrm{~J} 04442713+2512164$ & $0.6 \pm 0.6$ & $5.9 \pm 3.6$ & $0.98 \pm 0.03$ & $9 \pm 2$ & 1 \\
\hline 25 & ${ }^{\dagger}$ DQ Tau & $0.97 \pm 0.11$ & $83.1 \pm 2.8$ & $1.7 \pm 0.6$ & $161 \pm 86$ & 3,4 \\
\hline 26 & DR Tau & $49 \pm 65$ & $159 \pm 11$ & $103 \pm 31$ & $533 \pm 7$ & 5,8 \\
\hline 27 & ${ }^{\dagger} \mathrm{DS}$ Tau & $3.7 \pm 4.3$ & $19.94 \pm 0.25$ & 6.69 & $43 \pm 1.4$ & 9,2 \\
\hline 28 & GM Aur & $19.41 \pm 0.11$ & $176 \pm 5.3$ & $29.0 \pm 8.7$ & $707 \pm 4$ & $11,4,8,12$ \\
\hline 29 & V836 Tau & $0.84 \pm 0.18$ & $24 \pm 2$ & $1.42 \pm 0.65$ & $42 \pm 23$ & 13 \\
\hline 30 & IRAS $05022+2527$ & $2.9 \pm 3.4$ & $35.22 \pm 0.26$ & 5.21 & $87 \pm 1.3$ & 9,2 \\
\hline 31 & HD 34282 & $4.2 \pm 0.1$ & $110 \pm 10$ & $7.7 \pm 2.2$ & $218 \pm 131$ & 2 \\
\hline 32 & HD 294268 & $<0.9$ & $5 \pm 0.8$ & $<1.5 \pm 1.8$ & $19.4 \pm 5.4$ & 52 \\
\hline 33 & V2731 Ori & $0.95 \pm 0.13$ & $10.7 \pm 0.8$ & $1.6 \pm 0.6$ & $32.6 \pm 4.8$ & 52 \\
\hline 34 & J05391573-0230568 & $<2.43$ & $8 \pm 1$ & $<4.3 \pm 1.9$ & $13 \pm 2.7$ & 52 \\
\hline 35 & V602 Ori & $0.34 \pm 0.08$ & $7.8 \pm 0.8$ & $0.5 \pm 0.3$ & $17.4 \pm 4.2$ & 52 \\
\hline 36 & V606 Ori & $0.36 \pm 0.07$ & $14.3 \pm 0.8$ & $0.6 \pm 0.2$ & $31.4 \pm 4.8$ & 52 \\
\hline 37 & TWA 34 & $0.34 \pm 0.03$ & $0.54 \pm 0.06$ & $0.55 \pm 0.17$ & $0.66 \pm 0.32$ & 15 \\
\hline 38 & TW Hya & $12.4 \pm 1$ & $540 \pm 30$ & $45 \pm 4$ & $1620 \pm 50$ & \\
\hline 39 & T Cha & $7 \pm 7$ & 81.8 & $12.46 \pm 0.11$ & $198 \pm 4$ & \\
\hline 40 & HD 135344 & $10 \pm 10$ & $153 \pm 75$ & $18.6 \pm 0.8$ & $314 \pm 4.5$ & 16,17 \\
\hline 41 & HD 142527 & $17.4 \pm 0.01$ & $1090 \pm 0.3$ & $34 \pm 10$ & $2700 \pm 1500$ & 18 \\
\hline
\end{tabular}

Notes. Objects are sorted first by category and then by increasing RA values are presented here as given in the literature. For some rare cases, there are no errorbars. Values in italics correspond to extrapolation from the other frequency. Values given with the symbol "<" followed by an errorbar (e.g. $<1.5 \pm 0.5$ ) correspond to sources observed with ALMA where confusion with surrounding molecular clouds partly affects the emission from the source. Upper limits are $3 \sigma .{ }^{(1)}$ The flux of AB Aur is contaminated by the envelope. We have estimated the emission of the disk to be $\approx 80 \mathrm{Jy} \mathrm{km} \mathrm{s}^{-1}$ from interferometric observations with PdBI. ${ }^{(\dagger)}$ Binary stars.

References. 1 - Ricci et al. (2014); 2 - this work; 3 - Williams \& Best (2014); 4 - Guilloteau et al. (2011); 5 - Andrews \& Williams (2005); 6 - Koerner \& Sargent (1995); 7 - Beckwith et al. (1990); 8 - Thi et al. (2001); 9 - Akeson \& Jensen (2014); 10 - Mannings \& Emerson (1994); 11 - Öberg et al. (2010); 12 - Andrews \& Williams (2007); 13 - Duvert et al. (2000); 14 - Ansdell et al. (2015); 15 - Rodriguez et al. (2015); 16 - Dent et al. (2005); 17 - Brown et al. (2009); 18 - Perez et al. (2015); 19 - Zuckerman et al. (1995); 20 - Natta et al. (2004b); 21 - Zhang et al. (2014); 22 - Tsukagoshi et al. (2014); 23 - Romero et al. (2012); 24 - Salyk et al. (2014); 25 - Andrews et al. (2009); 26 - Ricci et al. (2012); 27 - Kastner et al. (2008); 28 - Jensen et al. (1996); 29 - Chapillon et al. (2008); 30 - Isella et al. (2010); 31 - Banzatti et al. (2011); 32 - Panić et al. (2010); 33 - Walsh et al. (2014); 34 - Czekala et al. (2015); 35 - Rosenfeld et al. (2013); 36 - Isella et al. (2007); 37 - Raman et al. (2006); 38 - Hardy et al. (2015); 39 - Hughes et al. (2008); 40 - Sheret et al. (2004); 41 - Kóspál et al. (2013); 42 - Moór et al. (2013); 43 - Moór et al. (2015); 44 - Nilsson et al. (2010); 45 - Pericaud et al. (in prep.); 46 - Sylvester et al. (2001); 47 - Panić et al. (2013); 48 - MacGregor et al. (2015); 49 - Dent et al. (2014); 50 - Williams \& Andrews (2006); 51 - Caceres et al. (2015); 52 - Williams et al. (2013); 53 - Canovas et al. (2016); 54 - Lieman-Sifry et al. (2016). 
J. Péricaud et al.: The hybrid disks: a search and study to better understand evolution of disks

Table D.1. continued.

\begin{tabular}{|c|c|c|c|c|c|c|}
\hline ID & Name & $\begin{array}{l}\mathrm{CO} J=2 \rightarrow 1 \\
\left(\mathrm{Jy} \mathrm{km} \mathrm{s}^{-1}\right)\end{array}$ & $\begin{array}{l}\text { Continuum } \\
1.3 \mathrm{~mm}(\mathrm{mJy})\end{array}$ & $\begin{array}{l}\mathrm{CO} J=3 \rightarrow 2 \\
\quad\left(\mathrm{Jy} \mathrm{km} \mathrm{s}^{-1}\right)\end{array}$ & $\begin{array}{c}\text { Continuum } \\
0.8 \mathrm{~mm}(\mathrm{mJy})\end{array}$ & References \\
\hline 42 & HD 143006 & $4.28 \pm 0.04$ & $43 \pm 3$ & $3 \pm 1$ & $78 \pm 43$ & $19,20,16$ \\
\hline 43 & J16042165-2130284 & $11 \pm 11$ & $113 \pm 53$ & $21.4 \pm 0.2$ & $226 \pm 1$ & 21 \\
\hline 44 & Sz 91 & $4.93 \pm 0.02$ & $40 \pm 1$ & 8.55 & $34.5 \pm 2.9$ & $53,22,23$ \\
\hline 45 & $\dagger$ AS 205 A $(\mathrm{N})$ & $19.23 \pm 0.27$ & $377 \pm 2$ & 65 & $905 \pm 7$ & 24,25 \\
\hline 46 & ${ }^{\dagger} \mathrm{AS} 205 \mathrm{~B}(\mathrm{~S})$ & $3.02 \pm 0.22$ & $64 \pm 1$ & $5.4 \pm 1.8$ & $55 \pm 7$ & 24,25 \\
\hline 47 & GSS 26 & $>4$ & 176 & $>7.3 \pm 19.9$ & $364 \pm 267$ & 2 \\
\hline 48 & GSS 39 & $>25$ & 304 & $>49.9 \pm 2.1$ & $663 \pm 3$ & 2,25 \\
\hline 49 & WL 18 & $>0.3$ & 24 & $>0.5 \pm 1.7$ & $42 \pm 28$ & 2 \\
\hline 50 & WL 14 & $>0.2$ & $<0.2$ & $>0.3 \pm 1.7$ & $<0.2 \pm 1.2$ & 2 \\
\hline 51 & ISO-Oph 102 & $0.3 \pm 0.3$ & $2.9 \pm 1.2$ & $0.53 \pm 0.05$ & $4.1 \pm 0.2$ & 26 \\
\hline 52 & YLW 16c & $>1.6$ & 45 & $>2.8 \pm 1.8$ & $82 \pm 58$ & 2 \\
\hline 53 & ROX 25 & $>0.6$ & 29 & $>1.0 \pm 1.7$ & $51 \pm 35$ & 2 \\
\hline 54 & Flying Saucer & $>2.1$ & 32 & $>3.7 \pm 1.9$ & $57 \pm 39$ & 2 \\
\hline 55 & AS 209 & 7.4 & 300 & 16.5 & $577 \pm 3$ & \\
\hline 56 & ${ }^{\dagger} \mathrm{V} 4046 \mathrm{Sgr}$ & $11.38 \pm 0.16$ & $451 \pm 20$ & $22 \pm 7$ & $1020 \pm 600$ & 27,28 \\
\hline \multicolumn{7}{|c|}{ Herbig AeBe disks } \\
\hline 57 & $\dagger$ V892 Tau & $22 \pm 29$ & $293 \pm 167$ & $85.4 \pm 26$ & $638 \pm 54$ & 8,5 \\
\hline 58 & AB Aur & $80^{1}$ & $103 \pm 18$ & $153 \pm 4$ & $359 \pm 67$ & $2,5,16$ \\
\hline 59 & MWC 480 & $22 \pm 0.2$ & $289.3 \pm 2.5$ & $52 \pm 1.1$ & $626 \pm 336$ & $11,4,16$ \\
\hline 60 & MWC 758 & $7.9 \pm 8.9$ & $56 \pm 1$ & $15 \pm 2$ & $217 \pm 40$ & $29,16,30$ \\
\hline 61 & HD 245185 & $0.17 \pm 0.03$ & $29 \pm 6$ & $0.3 \pm 0.1$ & $74.1 \pm 4.2$ & 14 \\
\hline 62 & CQ Tau & $3.1 \pm 0.18$ & $162 \pm 2$ & $5.95 \pm 0.96$ & $421 \pm 9$ & $11,4,16,31$ \\
\hline 63 & HD 100546 & $49 \pm 62$ & $540 \pm 272$ & $158 \pm 24$ & 1240 & 32,33 \\
\hline 64 & ${ }^{\dagger} \mathrm{AK}$ Sco & $2.21 \pm 0.01$ & $32.65 \pm 0.07$ & $5.7 \pm 1.9$ & $52 \pm 38$ & 34 \\
\hline 65 & HD 163296 & $46 \pm 5$ & $705 \pm 12$ & $109 \pm 11$ & $1910 \pm 20$ & 35,36 \\
\hline 66 & HD 169142 & $2.72 \pm 0.7$ & $169 \pm 5$ & $32.6 \pm 2.5$ & $349 \pm 190$ & 37,16 \\
\hline \multicolumn{7}{|c|}{ WTTS disks } \\
\hline 67 & $\mathrm{~J} 04182147+1658470$ & $<0.09$ & $<0.436$ & $<0.14$ & $<0.53$ & 38 \\
\hline 68 & $\dagger \mathrm{J} 04192625+2826142$ & $<0.09$ & 0.533 & $<0.14$ & $0.66 \pm 0.38$ & 38 \\
\hline 69 & J04242321+2650084 & $<0.09$ & $<0.426$ & $<0.14$ & $<0.51$ & 38 \\
\hline 70 & J04314503+2859081 & $<0.09$ & $<0.435$ & $<0.14$ & $<0.53$ & 38 \\
\hline 71 & J04325323+1735337 & $<0.09$ & $<0.438$ & $<0.14$ & $<0.53$ & 38 \\
\hline 72 & J04330422+2921499 & $<0.09$ & $<0.431$ & $<0.14$ & $<0.52$ & 38 \\
\hline 73 & J04364912+2412588 & $<0.09$ & $<0.435$ & $<0.14$ & $<0.53$ & 38 \\
\hline 74 & J04403979+2519061 & $<0.09$ & $<0.431$ & $<0.14$ & $<0.52$ & 38 \\
\hline 75 & ${ }^{\dagger} \mathrm{J} 04420548+2522562$ & $<0.09$ & $<0.423$ & $<0.14$ & $<0.51$ & 38 \\
\hline 76 & J08413703-7903304 & $<0.09$ & $<0.476$ & $<0.14$ & $<0.58$ & 38 \\
\hline 77 & J08422372-7904030 & $<0.09$ & $<0.472$ & $<0.14$ & $<0.58$ & 38 \\
\hline 78 & J11073519-7734493 & $<0.09$ & $<0.541$ & $<0.14$ & $<0.67$ & 38 \\
\hline 79 & ${ }^{\dagger} \mathrm{J} 11124268-7722230$ & $<0.09$ & $<0.494$ & $<0.14$ & $<0.60$ & 38 \\
\hline 80 & ${ }^{\dagger} \mathrm{J} 16002612-4153553$ & $<0.09$ & 0.696 & $<0.14$ & $0.88 \pm 0.5$ & 38 \\
\hline 81 & J16010896-3320141 & $<0.09$ & $<0.442$ & $<0.14$ & $<0.53$ & 38 \\
\hline 82 & J16031181-3239202 & $<0.09$ & $<0.453$ & $<0.14$ & $<0.55$ & 38 \\
\hline 83 & ${ }^{\dagger} \mathrm{J} 16085553-3902339$ & $<0.09$ & 1.813 & $<0.14$ & $2.5 \pm 1.5$ & 38 \\
\hline 84 & $\dagger J 16124119-1924182$ & $<0.09$ & $<0.459$ & $<0.14$ & $<0.56$ & 38 \\
\hline 85 & ${ }^{\dagger} \mathrm{J} 16220961-1953005$ & $<0.09$ & $<0.483$ & $<0.14$ & $<0.59$ & 38 \\
\hline 86 & J16223757-2345508 & $<0.09$ & $<0.461$ & $<0.14$ & $<0.56$ & 38 \\
\hline 87 & J16251469-2456069 & $<0.09$ & $<0.453$ & $<0.14$ & $<0.55$ & 38 \\
\hline 88 & ${ }^{\dagger} \mathrm{J} 16275209-2440503$ & $<0.09$ & $<0.442$ & $<0.14$ & $<0.53$ & 38 \\
\hline 89 & ${ }^{\dagger} \mathbf{J} 19002906-3656036$ & $<0.09$ & 0.569 & $<0.14$ & $0.7 \pm 0.3$ & 38 \\
\hline 90 & $\dagger \mathrm{J} 19012901-3701484$ & $<0.09$ & $<0.465$ & $<0.14$ & $<0.56$ & 38 \\
\hline \multicolumn{7}{|c|}{ Hybrid disks } \\
\hline 91 & 49 Ceti & $2 \pm 0.3$ & $<2.1$ & $9.5 \pm 1.9$ & $8.2 \pm 1.9$ & 39,40 \\
\hline 92 & HD 21997 & $2.17 \pm 0.23$ & $<1$ & $2.52 \pm 0.27$ & $2.69 \pm 0.3$ & $41,2,42$ \\
\hline 93 & HD 131835 & $0.798 \pm 0.035$ & $5.6 \pm 4.9$ & $2.74 \pm 0.55$ & $8.5 \pm 4.4$ & $54,43,44$ \\
\hline 94 & HIP 76310 & $1,41 \pm 0.08$ & $1.2 \pm 0.2$ & $2.4 \pm 0.5$ & $1.6 \pm 1.1$ & 54 \\
\hline 95 & HD 141569 & $9.57 \pm 0.03$ & $3.7 \pm 0.4$ & $14.6 \pm 1$ & $5.3 \pm 0.6$ & $45,16,46$ \\
\hline 96 & HIP 84881 & $1.18 \pm 0.04$ & $0.72 \pm 0.11$ & $2.5 \pm 1$ & $0.7 \pm 0.4$ & 54 \\
\hline
\end{tabular}


Table D.1. continued.

\begin{tabular}{|c|c|c|c|c|c|c|}
\hline ID & Name & $\begin{array}{l}\mathrm{CO} J=2 \rightarrow 1 \\
\quad\left(\mathrm{Jy} \mathrm{km} \mathrm{s}^{-1}\right)\end{array}$ & $\begin{array}{l}\text { Continuum } \\
1.3 \mathrm{~mm}(\mathrm{mJy})\end{array}$ & $\begin{array}{l}\mathrm{CO} J=3 \rightarrow 2 \\
\left(\mathrm{Jy} \mathrm{km} \mathrm{s}^{-1}\right)\end{array}$ & $\begin{array}{c}\text { Continuum } \\
0.8 \mathrm{~mm}(\mathrm{mJy})\end{array}$ & References \\
\hline \multicolumn{7}{|c|}{ Debris disks } \\
\hline 97 & HD 225200 & $<1.7 \pm 0.5$ & - & $<3.03$ & - & 2 \\
\hline 98 & ${ }^{\dagger} \mathrm{HD} 2772$ & $<0.84$ & - & $<1.4 \pm 1.8$ & - & 2 \\
\hline 99 & HD 14055 & $<0.47$ & $4.3 \pm 2.4$ & $<0.8 \pm 1.7$ & $6.4 \pm 1.1$ & 2,47 \\
\hline 100 & HD 15115 & $<2.48$ & $2.6 \pm 0.6$ & $<4.4 \pm 1.9$ & $8.5 \pm 1.2$ & $2,48,47$ \\
\hline 101 & HD 17848 & $<5.4 \pm 0.5$ & - & $<10.06$ & - & 2 \\
\hline 102 & ${ }^{\dagger} \mathrm{HD} 21620$ & $<0.91$ & - & $<1.5 \pm 1.8$ & - & 2 \\
\hline 103 & ${ }^{\dagger} \mathrm{HD} 23642$ & $0.710 \pm 0.18$ & - & $<1.4 \pm 1.8$ & - & 2 \\
\hline 104 & HD 24966 & $<1.6 \pm 0.5$ & - & $<2.75$ & - & 2 \\
\hline 105 & HD 30422 & $<1.2 \pm 0.5$ & - & $<2.13$ & - & 2 \\
\hline 106 & HD 31295 & $<1.1$ & - & $<1.9 \pm 1.8$ & - & 2 \\
\hline 107 & HD 35850 & $<1.3 \pm 0.5$ & - & $<2.17$ & $<5.4$ & 2 \\
\hline 108 & HD 38206 & $<1.5 \pm 0.5$ & - & $<2.67$ & - & 2 \\
\hline 109 & $\beta \mathrm{Pic}$ & $3.6 \pm 3.9$ & $34 \pm 18$ & $6.59 \pm 0.69$ & $60 \pm 6$ & 49 \\
\hline 110 & HD 42111 & $<1.21$ & - & $<2.1 \pm 1.8$ & - & 2 \\
\hline 111 & HD 54341 & $<1.4 \pm 0.5$ & - & $<2.39$ & - & 2 \\
\hline 112 & HD 71043 & $<1.5 \pm 0.5$ & - & $<2.64$ & - & 2 \\
\hline 113 & HD 71155 & $<1.5 \pm 0.5$ & - & $<2.56$ & - & 2 \\
\hline 114 & HD 78702 & $<1.2 \pm 0.5$ & $0.3 \pm 1.9$ & $<2.03$ & $0.3 \pm 2.3$ & 2 \\
\hline 115 & HD 136246 & $<1.4 \pm 0.5$ & - & $<2.37$ & - & 2 \\
\hline 116 & ${ }^{\dagger} \mathrm{HD} 159082$ & $<0.66$ & - & $<1.1 \pm 1.8$ & - & 2 \\
\hline 117 & HD 164249 & $<1.3 \pm 0.5$ & - & $<2.29$ & - & 2 \\
\hline 118 & HD 166191 & $<1.5 \pm 0.5$ & - & $<2.66$ & - & 2 \\
\hline 119 & HD 181296 & $<1.2 \pm 0.5$ & $9.1 \pm 3.7$ & $<2.11$ & 14.4 & 2 \\
\hline 120 & HD 182681 & $<1.6 \pm 0.5$ & $3.4 \pm 2.1$ & $<2.79$ & $5 \pm 1.3$ & 2,47 \\
\hline 121 & HD 218396 & $<0.5$ & 20 & $<0.8 \pm 1.7$ & $10.3 \pm 1.8$ & 2,50 \\
\hline 122 & HD 220825 & $<1.3 \pm 0.5$ & - & $<2.2$ & - & 2 \\
\hline
\end{tabular}


J. Péricaud et al.: The hybrid disks: a search and study to better understand evolution of disks

Table D.2. Stellar parameters. The classification is the same as in Table 2 .

\begin{tabular}{|c|c|c|c|c|c|c|c|c|}
\hline ID & Name & $\begin{array}{l}\text { Distance } \\
(\mathrm{pc})\end{array}$ & $\begin{array}{c}\text { Spectral } \\
\text { type }\end{array}$ & $\begin{array}{c}M_{\star} \\
\left(M_{\odot}\right) \\
\end{array}$ & $\begin{array}{c}L_{\star} \\
\left(L_{\odot}\right)\end{array}$ & $\begin{array}{c}\dot{M}_{\mathrm{acc}} \\
\left(M_{\odot} / \mathrm{yr}\right)\end{array}$ & $\begin{array}{l}\text { Age } \\
\text { Myr }\end{array}$ & References \\
\hline 1 & CIDA 1 & 140 & M5.5 & 0.13 & 0.13 & - & 2.7 & 1,2 \\
\hline 2 & IRAS 04113+2758 & 140 & - & - & - & - & - & \\
\hline 3 & CY Tau & 140 & M1.5 & 0.44 & 0.40 & $4.08 \times 10^{-9}$ & 5 & $1,2,3$ \\
\hline 4 & FQ Tau & 140 & M3 & 0.29 & 0.09 & $3.55 \times 10^{-7}$ & 6.8 & $4,2,5$ \\
\hline 5 & BP Tau & 76.8 & K7 & 0.79 & 0.97 & $4.08 \times 10^{-9}$ & 1.7 & $6,2,3$ \\
\hline 6 & RY Tau & 192 & K1 & 2.3 & 21.9 & $2.24 \times 10^{-8}$ & 3.86 & $6,7,8$ \\
\hline 7 & FV Tau A & 140 & M5 & 1.23 & 2.3 & $4.79 \times 10^{-8}$ & 3.7 & $4,2,9$ \\
\hline 8 & FW Tau c & 140 & M9 & 0.001 & - & $1.00 \times 10^{-11}$ & 2 & 48,49 \\
\hline 9 & FX Tau & 140 & M1 & 0.48 & 0.52 & $2.23 \times 10^{-9}$ & 1.7 & $10,2,5$ \\
\hline 10 & DK Tau & 140 & K8 & 0.71 & 1.32 & $1.05 \times 10^{-9}$ & 1 & $10,2,3$ \\
\hline 11 & HK Tau A & 140 & M0.5 & 0.54 & 0.44 & $1.95 \times 10^{-9}$ & 2.5 & $1,2,3$ \\
\hline 12 & HK Tau B & 140 & M2 & 0.37 & 0.03 & - & 4.1 & 1,2 \\
\hline 13 & V710 Tau & 140 & M0.5 & 0.54 & 0.57 & - & 1.7 & 1,2 \\
\hline 14 & GG Tau & 140 & K7 & 1.35 & 0.85 & $3.02 \times 10^{-9}$ & 2.1 & $11,2,9$ \\
\hline 15 & GK Tau A & 140 & K7 & 0.78 & 1.35 & $6.45 \times 10^{-9}$ & 1.1 & 5,2 \\
\hline 16 & DL Tau & 140 & K7 & 0.81 & 0.74 & $3.22 \times 10^{-8}$ & 2.6 & $1,2,3$ \\
\hline 17 & HN Tau & 140 & K5 & 0.91 & 0.42 & $2.72 \times 10^{-9}$ & 5.4 & $12,2,3$ \\
\hline 18 & DM Tau & 140 & M1 & 0.47 & 0.23 & $2.25 \times 10^{-9}$ & 5 & $5,2,3$ \\
\hline 19 & IT Tau B & 140 & K3 & 1.41 & 1.43 & - & 4.6 & 10,2 \\
\hline 20 & AA Tau & 140 & K7 & 0.79 & 0.87 & $3.91 \times 10^{-9}$ & 2 & $5,2,3$ \\
\hline 21 & HO Tau & 140 & M0.5 & 0.56 & 0.13 & - & 13.2 & 1,2 \\
\hline 22 & hbc 411 & 140 & - & - & - & - & - & \\
\hline 23 & LkCa 15 & 140 & K5 & 1.05 & 0.81 & $6.77 \times 10^{-10}$ & 3.7 & $5,2,3$ \\
\hline 24 & J04442713+2512164 & 140 & M7.3 & - & - & - & - & \\
\hline 25 & DQ Tau & 140 & M0 & 1.27 & 0.4 & $2.53 \times 10^{-9}$ & 3.5 & $13,14,2,3$ \\
\hline 26 & DR Tau & 140 & K5 & 1.2 & 1.82 & $1.42 \times 10^{-8}$ & 1.5 & $1,2,3$ \\
\hline 27 & DS Tau & 140 & K5 & 1.05 & 0.76 & $3.35 \times 10^{-8}$ & 4.1 & $1,2,3$ \\
\hline 28 & GM Aur & 140 & K3 & 1.35 & 1.23 & $1.21 \times 10^{-8}$ & 5.6 & $15,2,3$ \\
\hline 29 & V836 Tau & 140 & K7 & 0.83 & 0.57 & $4.20 \times 10^{-9}$ & 3.7 & $1,2,3$ \\
\hline 30 & IRAS $05022+2527$ & 140 & M0 & - & - & - & - & \\
\hline 31 & HD 34282 & 350 & A0 & 2.35 & 5.13 & $<1.95 \times 10^{-8}$ & 6.5 & $50,19,40$ \\
\hline 32 & HD 294268 & 420 & F8 & - & - & - & - & 53 \\
\hline 33 & V2731 Ori & 420 & - & - & - & - & - & \\
\hline 34 & J05391573-0230568 & 420 & K9 & - & - & - & - & 53 \\
\hline 35 & V602 Ori & 420 & M3 & - & - & - & - & 53 \\
\hline 36 & V606 Ori & 420 & - & - & - & - & - & \\
\hline 37 & TWA 34 & 47 & M4.9 & - & 0.014 & - & 6 & 16,17 \\
\hline 38 & TW Hya & 54 & M2.5 & 0.4 & 0.19 & $4.75 \times 10^{-9}$ & 3 & 18,3 \\
\hline 39 & T Cha & 164 & K0 & 1.5 & - & - & - & \\
\hline 40 & HD 135344 & 140 & F3 & 1.5 & 7.08 & $4.27 \times 10^{-8}$ & 8 & 19,20 \\
\hline 41 & HD 142527 & 140 & F6 & 1.6 & 7.94 & $3.55 \times 10^{-8}$ & 8.8 & 19,20 \\
\hline
\end{tabular}

References. 1 - Luhman et al. (2010); 2 - Andrews et al. (2013); 3 - Rigliaco et al. (2015); 4 - Hartigan \& Kenyon (2003); 5 - Hartmann et al. (1998); 6 - Hartigan et al. (1995); 7 - Hernández et al. (2004); 8 - Mendigutía et al. (2011); 9 - White \& Ghez (2001); 10 - Duchêne et al. (1999); 11 - White et al. (1999); 12 - Hartigan et al. (1994); 13 - Mathieu et al. (1997); 14 - Czekala et al. (2016); 15 - Espaillat et al. (2010); 16 - Schneider et al. (2012); 17 - Herczeg \& Hillenbrand (2014); 18 - Vacca \& Sandell (2011); 19 - Garcia Lopez et al. (2006); 20 - Fairlamb et al. (2015); 21 - Natta et al. (2004b); 22 - Bast et al. (2011); 23 - Salyk et al. (2013); 24 - Andrews et al. (2009); 25 - Ricci et al. (2010); 26 - Reboussin et al. (2015); 27 - Natta et al. (2006); 28 - Isella et al. (2009); 29 - Wilking et al. (2005); 30 - Andrews et al. (2010); 31 - Natta et al. (2004a); 32 - Grosso et al. (2003); 33 - Herbig \& Bell (1988); 34 - Mora et al. (2001); 35 - Beskrovnaya et al. (1999); 36 - Wade et al. (2007); 37 - Donehew \& Brittain (2011); 38 - Meeus et al. (2012); 39 - van Boekel et al. (2005); 40 - Manoj et al. (2006); 41 - Czekala et al. (2015); 42 - Hardy et al. (2015); 43 - Cieza et al. (2012); 44 - Montesinos et al. (2009); 45 - Torres et al. (2006); 46 - Pecaut et al. (2012); 47 - Binks \& Jeffries (2014); 48 - Kraus et al. (2014); 49 - Bowler et al. (2014); 50 - Piétu et al. (2003); 51 - Lieman-Sifry et al. (2016); 52 - Hernández et al. (2005); 53 - Williams et al. (2013). No reference means data comes from SIMBAD. 
Table D.2. continued.

\begin{tabular}{|c|c|c|c|c|c|c|c|c|}
\hline ID & Name & $\begin{array}{l}\text { Distance } \\
\quad(\mathrm{pc})\end{array}$ & $\begin{array}{l}\text { Spectral } \\
\text { type }\end{array}$ & $\begin{array}{c}M_{\star} \\
\left(M_{\odot}\right)\end{array}$ & $\begin{array}{l}L_{\star} \\
\left(L_{\odot}\right)\end{array}$ & $\begin{array}{c}\dot{M}_{\mathrm{acc}} \\
\left(M_{\odot} / \mathrm{yr}\right)\end{array}$ & $\begin{array}{l}\text { Age } \\
\text { Myr }\end{array}$ & References \\
\hline 42 & HD 143006 & 82 & G6 & 1 & 0.8 & $5.11 \times 10^{-9}$ & $>1000$ & 21,3 \\
\hline 43 & $\mathrm{~J} 16042165-2130284$ & 145 & - & - & - & - & - & \\
\hline 44 & $\mathrm{Sz} 91$ & 200 & M2 & - & 0.25 & - & - & 17 \\
\hline 45 & AS 205 A $(\mathrm{N})$ & 125 & K7 & 1.5 & 7.1 & $7.94 \times 10^{-8}$ & 1.6 & $22,23,24$ \\
\hline 46 & AS 205 B (S) & 125 & M0.1 & 0.3 & 2.19 & $2.09 \times 10^{-7}$ & - & 17,23 \\
\hline 47 & GSS 26 & 135 & M0 & 0.56 & 1.39 & - & 0.5 & 25,26 \\
\hline 48 & GSS 39 & 135 & M1 & 0.6 & 1 & $3.70 \times 10^{-8}$ & 1 & $27,24,28$ \\
\hline 49 & WL 18 & 135 & K7 & - & 0.3 & - & - & 29,30 \\
\hline 50 & WL 14 & 135 & - & - & - & - & - & \\
\hline 51 & ISO-Oph 102 & 135 & M6 & 0.06 & 0.08 & $1.00 \times 10^{-9}$ & - & 31 \\
\hline 52 & YLW 16c & 135 & M1 & 0.48 & 1.11 & - & 0.5 & 25,26 \\
\hline 53 & ROX 25 & 135 & - & - & - & - & - & \\
\hline 54 & Flying Saucer & 120 & M1 & - & 0.14 & - & - & 32 \\
\hline 55 & AS 209 & 125 & K5 & 1.4 & 2.5 & $3.02 \times 10^{-8}$ & 0.5 & $33,23,24$ \\
\hline 56 & V4046 Sgr & 54 & - & - & - & - & - & \\
\hline \multicolumn{9}{|c|}{ Herbig AeBe disks } \\
\hline 57 & V892 Tau & 140 & B8 & 2.8 & 38.8 & - & 46 & 7,2 \\
\hline 58 & AB Aur & 139 & A1 & 2.6 & 51.3 & $1.41 \times 10^{-7}$ & 3.94 & 7,19 \\
\hline 59 & MWC 480 & 146 & A 4.5 & 1.91 & 19.1 & $<5.89 \times 10^{-8}$ & 7.1 & $34,2,8$ \\
\hline 60 & MWC 758 & 279 & A3 & 1.8 & 17.4 & $8.90 \times 10^{-7}$ & 3.5 & $35,36,37,38$ \\
\hline 61 & HD 245185 & 450 & A1 & 2.2 & 22.9 & - & 7.97 & 7 \\
\hline 62 & CQ Tau & 113 & F3 & 1.6 & 7.1 & $<5.01 \times 10^{-9}$ & 13.27 & 7,8 \\
\hline 63 & HD 100546 & 97 & B9 & 2.5 & 40.7 & $9.12 \times 10^{-8}$ & 3.46 & $39,40,20$ \\
\hline 64 & AK Sco & 103 & F5 & 2.49 & - & - & - & 41 \\
\hline 65 & HD 163296 & 119 & A1 & 2.49 & 33.9 & $3.23 \times 10^{-8}$ & 3 & $19,40,20$ \\
\hline 66 & HD 169142 & 145 & A5 & 2 & 18.2 & $3.98 \times 10^{-8}$ & 5.42 & 19,40 \\
\hline \multicolumn{9}{|c|}{ WTTS disks } \\
\hline 67 & J04182147+1658470 & 135 & K5 & - & - & - & & \\
\hline 68 & $\mathrm{~J} 04192625+2826142$ & 135 & K7 & 0.67 & - & $<1.00 \times 10^{-11}$ & 3.5 & 42,43 \\
\hline 69 & $\mathrm{~J} 04242321+2650084$ & 135 & M2 & 0.35 & - & $<1.00 \times 10^{-11}$ & 6.7 & 42,43 \\
\hline 70 & $\mathrm{~J} 04314503+2859081$ & 135 & F5 & - & - & $<1.00 \times 10^{-11}$ & - & 42,43 \\
\hline 71 & J04325323+1735337 & 135 & M2 & 0.36 & - & - & 3.1 & 42 \\
\hline 72 & J04330422+2921499 & 135 & B9 & - & - & $<1.00 \times 10^{-11}$ & - & 42,43 \\
\hline 73 & $\mathrm{~J} 04364912+2412588$ & 135 & $\mathrm{~F} 2$ & - & - & $<1.00 \times 10^{-11}$ & - & 42,43 \\
\hline 74 & J04403979+2519061 & 135 & M5 & 0.17 & - & $<1.00 \times 10^{-11}$ & - & 42 \\
\hline 75 & $\mathrm{~J} 04420548+2522562$ & 135 & K7 & 0.65 & - & - & - & 42 \\
\hline 76 & J08413703-7903304 & 97 & M3 & 0.27 & - & - & 3.4 & 42 \\
\hline 77 & J08422372-7904030 & 97 & M3 & 0.31 & - & - & 15 & 42 \\
\hline 78 & J11073519-7734493 & 160 & M4 & 0.20 & - & - & 12.4 & 42 \\
\hline 79 & $\mathrm{~J} 11124268-7722230$ & 160 & G8 & 1.4 & - & - & 6.9 & 42 \\
\hline 80 & $\mathrm{~J} 16002612-4153553$ & 150 & M5.3 & 0.17 & - & - & 26.3 & 42 \\
\hline 81 & J16010896-3320141 & 150 & G8 & 1.1 & - & - & 7.7 & 42 \\
\hline 82 & J16031181-3239202 & 150 & K7 & 0.7 & - & - & 2.6 & 42 \\
\hline 83 & $\mathrm{~J} 16085553-3902339$ & 200 & M6 & 0.1 & - & - & 6.6 & 42 \\
\hline 84 & J16124119-1924182 & 119 & K8 & 0.64 & - & - & 2.2 & 42 \\
\hline 85 & $\mathrm{~J} 16220961-1953005$ & 119 & M3.7 & 0.34 & - & - & 9.9 & 42 \\
\hline 86 & $\mathrm{~J} 16223757-2345508$ & 119 & M2.5 & 0.33 & - & - & 3 & 42 \\
\hline 87 & J16251469-2456069 & 119 & M0 & 0.56 & - & - & 4.2 & 42 \\
\hline 88 & $\mathrm{~J} 16275209-2440503$ & 129 & K7 & 0.77 & - & - & 4.7 & 42 \\
\hline 89 & J19002906-3656036 & 129 & M4 & 0.24 & - & - & 10.2 & 42 \\
\hline 90 & J19012901-3701484 & 116 & M3.8 & 0.21 & - & - & - & 42 \\
\hline \multicolumn{9}{|c|}{ Hybrid disks } \\
\hline 91 & 49 Ceti & 59 & A1 & 2.17 & 21.8 & - & 8.9 & 44 \\
\hline 92 & HD 21997 & 72 & A3 & 1.8 & - & - & 30 & 45 \\
\hline 93 & HD 131835 & 122 & $\mathrm{~A} 2$ & - & - & - & 16 & 46 \\
\hline 94 & HIP 76310 & 151 & A0 & 2.2 & 26.9 & - & 10 & 52,51 \\
\hline 95 & HD 141569 & 116 & A0 & 2.18 & 22.9 & $2.24 \times 10^{-8}$ & 6.65 & $34,40,20$ \\
\hline 96 & HIP 84881 & 118 & A0 & 2.9 & 15 & - & 10 & 51 \\
\hline
\end{tabular}


J. Péricaud et al.: The hybrid disks: a search and study to better understand evolution of disks

Table D.2. continued.

\begin{tabular}{|c|c|c|c|c|c|c|c|c|}
\hline ID & Name & $\begin{array}{c}\text { Distance } \\
(\mathrm{pc})\end{array}$ & $\begin{array}{c}\text { Spectral } \\
\text { type }\end{array}$ & $\begin{array}{c}M_{\star} \\
\left(M_{\odot}\right)\end{array}$ & $\begin{array}{c}L_{\star} \\
\left(L_{\odot}\right)\end{array}$ & $\begin{array}{c}\dot{M}_{\mathrm{acc}} \\
\left(M_{\odot} / \mathrm{yr}\right)\end{array}$ & $\begin{array}{l}\text { Age } \\
\text { Myr }\end{array}$ & References \\
\hline \multicolumn{9}{|c|}{ Debris disks } \\
\hline 97 & HD 225200 & 129 & $\mathrm{~A} 0$ & - & - & - & - & \\
\hline 98 & HD 2772 & 116 & B8 & - & - & - & - & \\
\hline 99 & HD 14055 & 34 & A1 & - & - & - & - & \\
\hline 100 & HD 15115 & 45 & $\mathrm{~F} 2$ & - & - & - & - & \\
\hline 101 & HD 17848 & 51 & $\mathrm{~A} 2$ & - & - & - & - & \\
\hline 102 & HD 21620 & 149 & A0 & - & - & - & - & \\
\hline 103 & HD 23642 & 139 & A0 & - & - & - & - & \\
\hline 104 & HD 24966 & 104 & A0 & - & - & - & - & \\
\hline 105 & HD 30422 & 56 & A3 & - & - & - & - & \\
\hline 106 & HD 31295 & 36 & A0 & - & - & - & - & \\
\hline 107 & HD 35850 & 27 & $\mathrm{~F} 8$ & 1.2 & 1.78 & $2.41 \times 10^{-12}$ & - & 3 \\
\hline 108 & HD 38206 & 69 & A0 & - & - & - & - & \\
\hline 109 & $\beta \mathrm{Pic}$ & 19 & A6 & - & - & - & 20 & 47 \\
\hline 110 & HD 42111 & 201 & A3 & - & - & - & - & \\
\hline 111 & HD 54341 & 102 & A0 & - & - & - & - & \\
\hline 112 & HD 71043 & 73 & $\mathrm{~A} 0$ & - & - & - & - & \\
\hline 113 & HD 71155 & 38 & A0 & - & - & - & - & \\
\hline 114 & HD 78702 & 84 & A0 & - & - & - & - & \\
\hline 115 & HD 136246 & 144 & A1 & - & - & - & - & \\
\hline 116 & HD 159082 & 135 & B9 & - & - & - & - & \\
\hline 117 & HD 164249 & 48 & F6 & - & - & - & - & \\
\hline 118 & HD 166191 & 119 & F8 & - & - & - & - & \\
\hline 119 & HD 181296 & 48 & A0 & - & - & - & - & \\
\hline 120 & HD 182681 & 69 & B9 & - & - & - & - & \\
\hline 121 & HD 218396 & 39 & A5 & - & - & - & - & \\
\hline 122 & HD 220825 & 47 & $\mathrm{~A} 2$ & - & - & - & - & \\
\hline
\end{tabular}

